\title{
Combined Impact of Denticity and Orientation on Molecular-Scale Charge Transport
}

\section{Paris Yasini ${ }^{1}$, Stuart Shepard ${ }^{2}$, Tim Albrecht ${ }^{3}$, Manuel Smeu ${ }^{* 2}$, and Eric Borguet $^{* 1}$}

\footnotetext{
${ }^{1}$ Department of Chemistry, Temple University, Philadelphia, PA 19122, USA

${ }^{2}$ Department of Physics, Binghamton University, Binghamton, NY 13902, USA

${ }^{3}$ School of Chemistry, University of Birmingham, Birmingham, B15 2TT, UK
}

\section{Contents:}

1- Solution and materials

a. Chemicals

b. Electrochemical cell preparation

2- Cyclic voltammetry

3- In situ-scanning tunneling microscopy (STM)

a. Detailed analysis of STM images of TCNQ

b. STM images of TCNQ vs sample potential

c. Detailed analysis of STM images of $\mathrm{F}_{4} \mathrm{TCNQ}$

d. STM images of $\mathrm{F}_{4}$ TCNQ $v s$ sample potential

4- In situ-scanning tunneling microscopy-break junction (STM-BJ)

a. Method

b. Data selection criteria

5- MPVC analysis

a. Description, method and data analysis

b. Comparison of manual selection vs MPVC classification of low-conductance data

6- STM-BJ control experiments

7- Single molecule conductance vs sample bias

8- DFT calculations
a. Method
b. $\mathrm{F}_{4} \mathrm{TCNQ}-$ Geometries and Transmission
c. TCNQ - Geometries and Transmission
d. Calculations of isolated $\mathrm{F}_{4} \mathrm{TCNQ}$ and TCNQ molecules 


\section{1- Solutions and materials:}

\section{a. Chemicals:}

7,7,8,8-tetracyanoquinodimethane

(TCNQ),

2,3,5,6-tetrafluoro-7,7,8,8,-

tetracyanoquinodimethane ( $\mathrm{F}_{4} \mathrm{TCNQ}$ ) and $\mathrm{H}_{2} \mathrm{SO}_{4}$ (Trace Metal Analysis grade, 99.999\%) were purchased from Sigma-Aldrich and used as received.

A single crystal $\mathrm{Au}$ (111) disk electrode (10 mm diameter, $2.0 \mathrm{~mm}$ thick, MaTeck, Germany) was used as a working electrode for STM imaging and break junction experiments. STM tips were prepared by electrochemically etching a $0.25 \mathrm{~mm}$ tungsten wire $(0.25 \mathrm{~mm}$ diameter, Premion, 99.95\%, Alfa Aesar) in $2 \mathrm{M} \mathrm{NaOH}$ solution using a platinum ring electrode. Tips for single molecule conductivity (SMC) measurements were prepared by electrochemically etching a gold wire (0.25 mm diameter, Premion, 99.998\%, Alfa Aesar) in a $\mathrm{C}_{2} \mathrm{H}_{5} \mathrm{OH}$ : $\mathrm{HCl}$ (1:1) solution. Platinum wires $(0.20 \mathrm{~mm}$ diameter, Alfa Aesar) were used as the reference and counter electrodes in electrochemical STM measurements. All the tips were coated with melted polyethylene to limit the faradaic current to less than $10 \mathrm{pA}$ at $0.1 \mathrm{~V}$ bias in an electrochemical environment.

\section{b. Electrochemical cell preparation:}

Prior to each experiment, the substrate, the Teflon cell, the O-ring as well as the reference and counter electrodes were cleaned by immersion in freshly prepared hot piranha solution $\left(1: 3 \mathrm{H}_{2} \mathrm{O}_{2}\right.$ (J. T. Baker, CMOS)/ $\mathrm{H}_{2} \mathrm{SO}_{4}(96 \%$, J. T. Baker, CMOS) for 1 h. (CAUTION: "piranha” solution is a very reactive mixture! It must be handled with great care; use of protective equipment, such as gloves, goggles, and lab coat, is necessary.) Extensive rinsing by ultra-pure deionized (DI) water produced by a Thermoscientific Barnstead Easypure II purification system equipped with a UV lamp (water resistivity $>18 \mathrm{M} \Omega . \mathrm{cm}$ ) was followed by boiling in DI water for 5 minutes. To avoid contamination while transferring these pieces for cell setup, the gold crystal, the O-ring and the Teflon holder were covered in DI water. A hydrogen flame was used to anneal the crystal, followed by quenching in hydrogen-saturated ultrapure DI water. Then the cell was quickly assembled into the electrochemical cell and $\mathrm{H}_{2} \mathrm{SO}_{4}(0.05 \mathrm{M})$ was added to cover the working $(\mathrm{Au}(111))$, reference and counter electrodes. Under potential control, two drops of saturated solution of target molecules were added to the STM cell containing the electrolyte. All potentials $\left(\mathrm{V}_{\mathrm{SCE}}\right)$ are reported with respect to the saturated calomel electrode. 


\section{2- Cyclic voltammetry:}

The electrochemical behavior of the $\mathrm{Au}(111)$ electrode in $0.05 \mathrm{M}$ sulfuric acid with and without the target molecules was investigated within the double layer region from -0.3 to $0.7 \mathrm{~V}_{\text {SCE. }}$ The sulfate ion adsorption peak observed at $0.7 \mathrm{~V}_{\mathrm{SCE}}$ in the $\mathrm{CV}$ of bare $\mathrm{Au}(111)$ (Figure S1-dashed line) indicates that the $\mathrm{Au}(111)$ surface is free of contamination. The observed reversible peaks at $0.2 \mathrm{~V}_{\mathrm{SCE}}$ correspond to the lifting and recovery of the $\mathrm{Au}(111)$ reconstruction as reported in previous studies ${ }^{1}$. Addition of TCNQ/F4TCNQ results in two reversible peaks in the $0.50-0.65$ $\mathrm{V}_{\mathrm{SCE}}$ range (Figure S1, red and green lines) as expected due to their similar backbone structure. Previous studies have shown that both TCNQ and F4TCNQ in acetonitrile undergo two electrochemically reversible one-electron reduction processes at potentials below $0.1 \mathrm{~V}_{\mathrm{SCE}}{ }^{2-3}$. Therefore, it appears that the observed reversible peaks, lying in the $0.50-0.65 \mathrm{~V}_{\mathrm{SCE}}$ range, are a result of the molecular order/disorder transition ${ }^{4}$, as confirmed using in situ STM images as described in the main text. The substrate reconstruction peaks in the presence of TCNQ shift to higher potential ( 0.26 vs. $\left.0.22 \mathrm{~V}_{\mathrm{SCE}}\right)$ whereas these two peaks disappeared in the presence of $\mathrm{F}_{4} \mathrm{TCNQ}$ implying different molecule-substrate interactions. These observations were confirmed by STM images of TCNQ and $\mathrm{F}_{4} \mathrm{TCNQ}$, discussed in the main text.

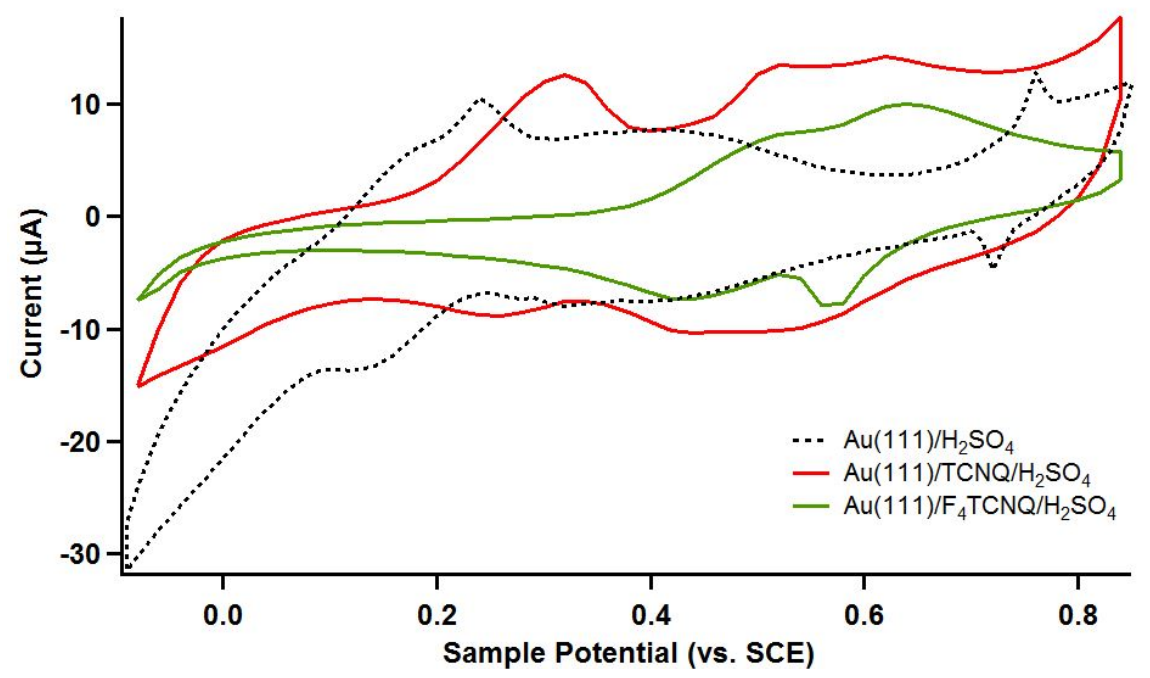

Figure- S1. Cyclic voltammograms of a freshly prepared, flame annealed bare $\mathrm{Au}$ (111) electrode in 0.05 $\mathrm{M} \mathrm{H}_{2} \mathrm{SO}_{4}$ aqueous electrolyte (Dashed line), $0.1 \mathrm{ml}$ saturated TCNQ added in $0.05 \mathrm{M} \mathrm{H}_{2} \mathrm{SO}_{4}$ aqueous electrolyte on $\mathrm{Au}(111)$ (Red line) and $0.1 \mathrm{ml}$ saturated $\mathrm{F}_{4} \mathrm{TCNQ}$ in $0.05 \mathrm{M} \mathrm{H}_{2} \mathrm{SO}_{4}$ aqueous electrolyte on $\mathrm{Au}(111)$. All voltammograms were acquired at a sweep rate of $100 \mathrm{mV} \cdot \mathrm{s}^{-1}$ 


\section{3- In situ-scanning tunneling microscopy (STM)}

STM images were obtained using a PicoScan STM system (Molecular Imaging). A $1 \mathrm{nA} / \mathrm{V}$ preamplifier was used for all STM imaging. In order to control the surface and tip potentials independently a PicoStat bipotentiostat was used. All the STM images were recorded in constant-current mode under surface potential control at room temperature.

Scanning tunneling microscopy break junction (STM-BJ) experiments: The STM-BJ experiments were carried out with a PicoScan STM system (Molecular Imaging).

\section{a. Detailed analysis of STM images of TCNQ}

(a)

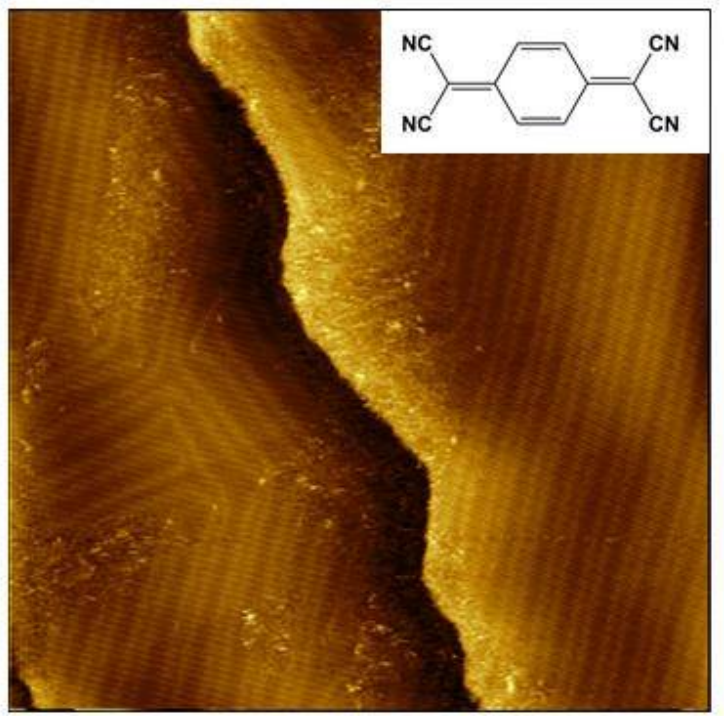

(c)

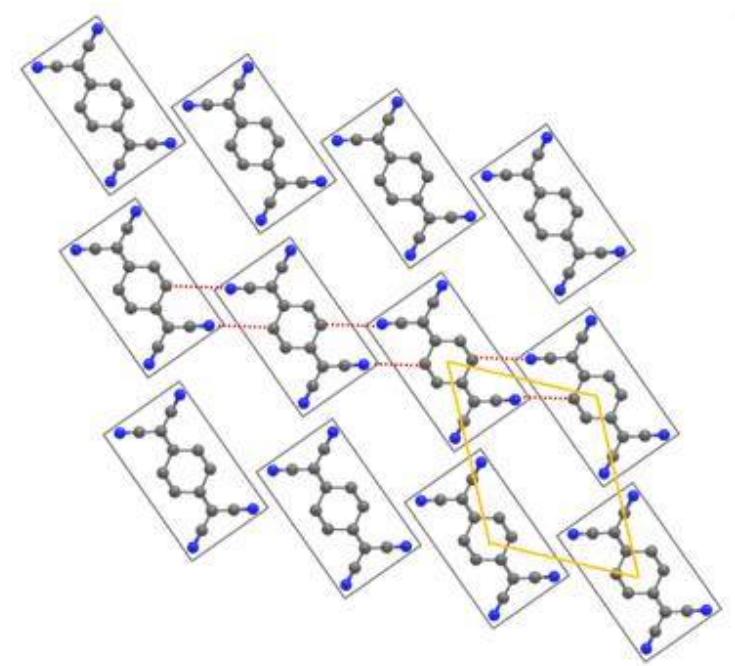

(b)

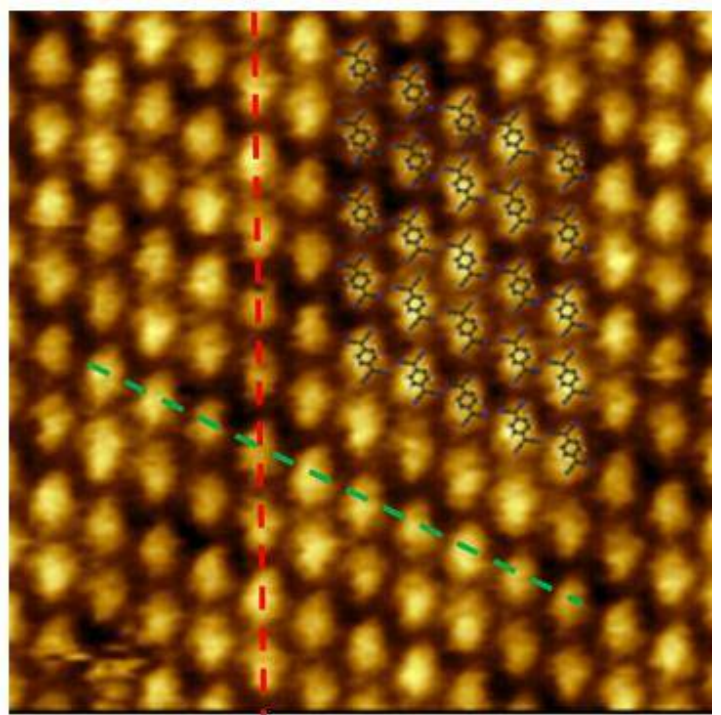

(d)
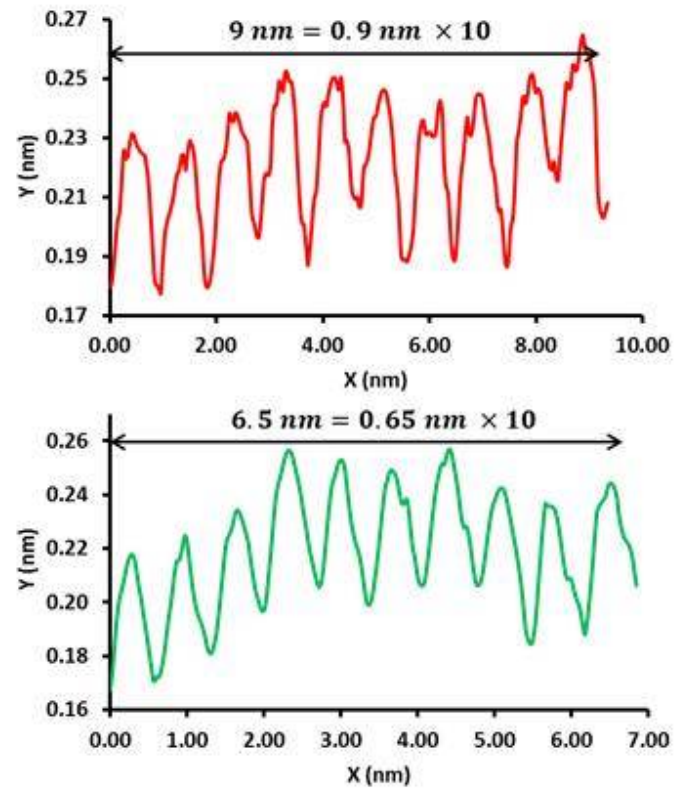

Figure S2. STM images of TCNQ/0.05 $\mathrm{M} \mathrm{H}_{2} \mathrm{SO}_{4}$ on $\mathrm{Au}(111)$ at $\mathrm{V}_{\mathrm{S}}=0 \mathrm{~V}_{\mathrm{SCE}}, \mathrm{V}_{\text {bias }}=0.03 \mathrm{~V}$ and $\mathrm{I}_{\mathrm{t}}=0.1$ $\mathrm{nA}$ (a) scan area $100 \times 100 \mathrm{~nm}^{2}$, inset: TCNQ structure (b) Scan area $10 \times 10 \mathrm{~nm}^{2}$, (c) Proposed molecular packing and unit cell containing one TCNQ molecule, (d) Cross sections of the ordered structure in the direction specified by the red and green lines in image (b). 


\section{b. STM images of TCNQ vs sample potential}

(a)

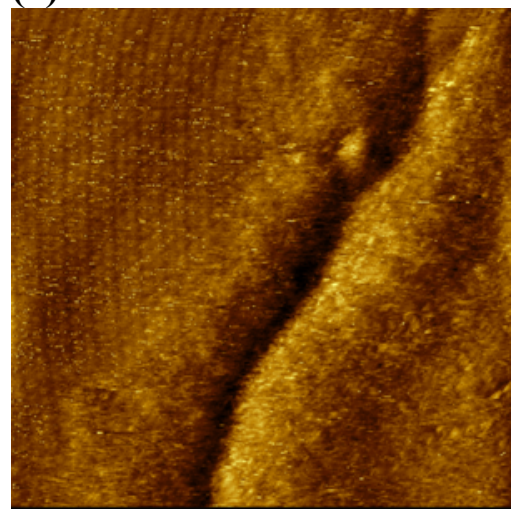

(d)

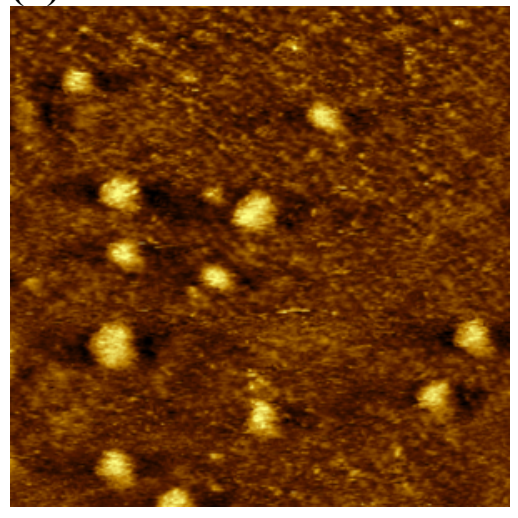

(b)

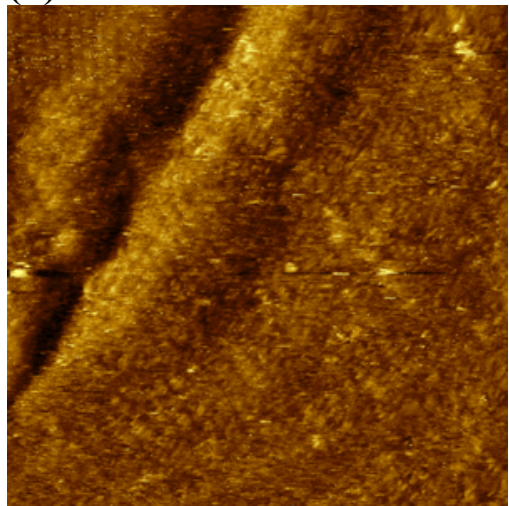

(e)

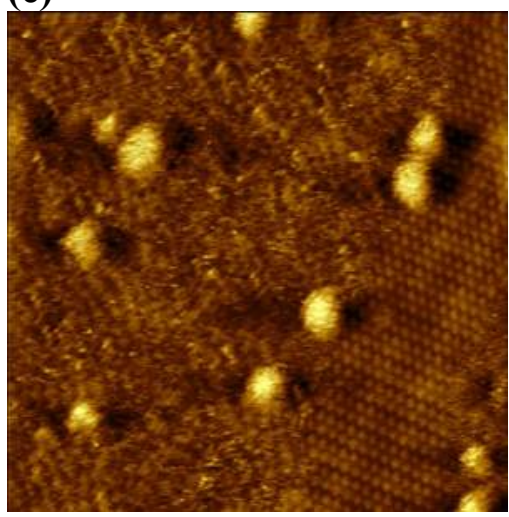

(c)

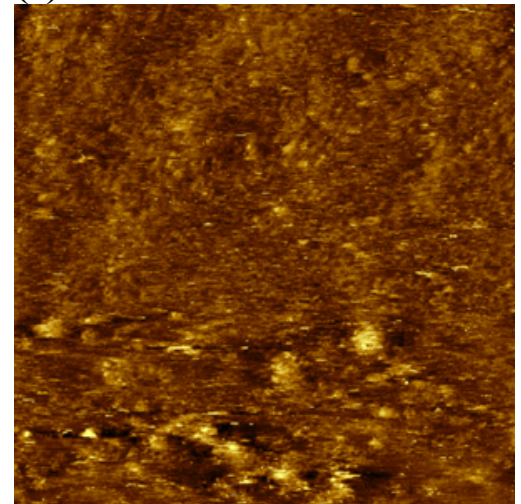

(f)

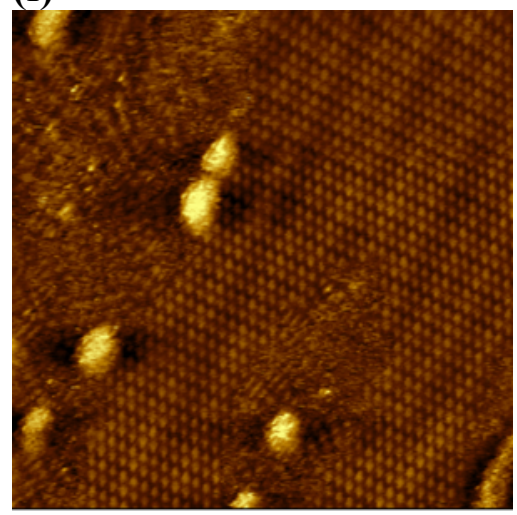

Figure S3. STM images of TCNQ/0.05 $\mathrm{M} \mathrm{H}_{2} \mathrm{SO}_{4}$ on $\mathrm{Au}(111), \mathrm{I}_{\mathrm{t}}=0.1 \mathrm{nA}$, scan area $30 \times 30 \mathrm{~nm}^{2}$ at (a) $\mathrm{V}_{\mathrm{S}}=0.15 \mathrm{~V}_{\mathrm{SCE}}$, (b) $\mathrm{V}_{\mathrm{S}}=0.30 \mathrm{~V}_{\mathrm{SCE}}$, (c) $\mathrm{V}_{\mathrm{S}}=0.50 \mathrm{~V}_{\mathrm{SCE}}$, (d) $\mathrm{V}_{\mathrm{S}}=0.70 \mathrm{~V}_{\mathrm{SCE}}$, (e) $\mathrm{V}_{\mathrm{S}}=0.20 \mathrm{~V}_{\mathrm{SCE}}$, (f) $\mathrm{V}_{\mathrm{S}}=0.00$ $\mathrm{V}_{\mathrm{SCE}}$

Consecutive STM images show the order/disorder phase transition upon moving the sample potential to more positive values. The ordered structure disappeared completely at $\mathrm{V}_{\mathrm{S}}=0.50$ $\mathrm{V}_{\mathrm{SCE}}$ and reappeared again at $\mathrm{V}_{\mathrm{S}}=0.20 \mathrm{~V}_{\mathrm{SCE}}$. 


\section{c. Detailed analysis of STM images of $\mathrm{F}_{4} \mathrm{TCNQ}$}

(a)

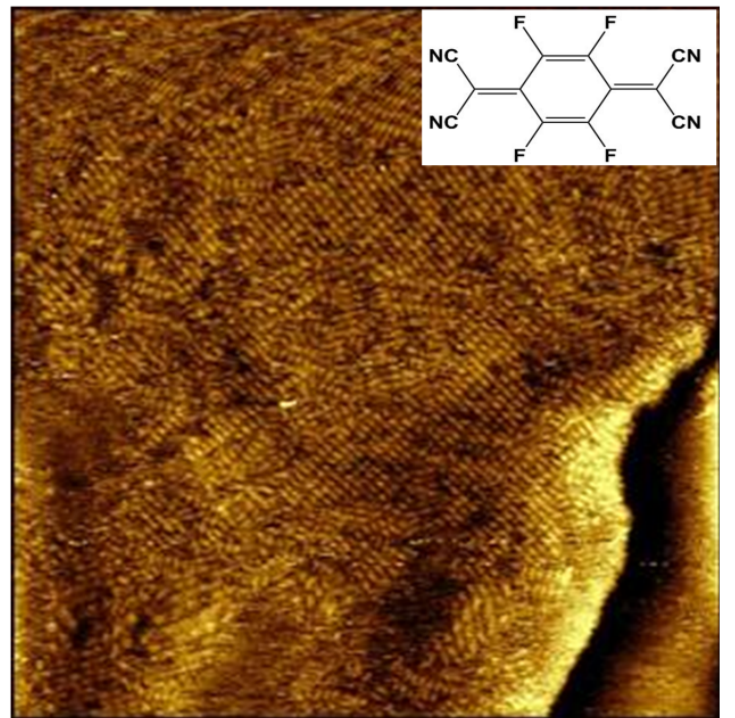

(c)

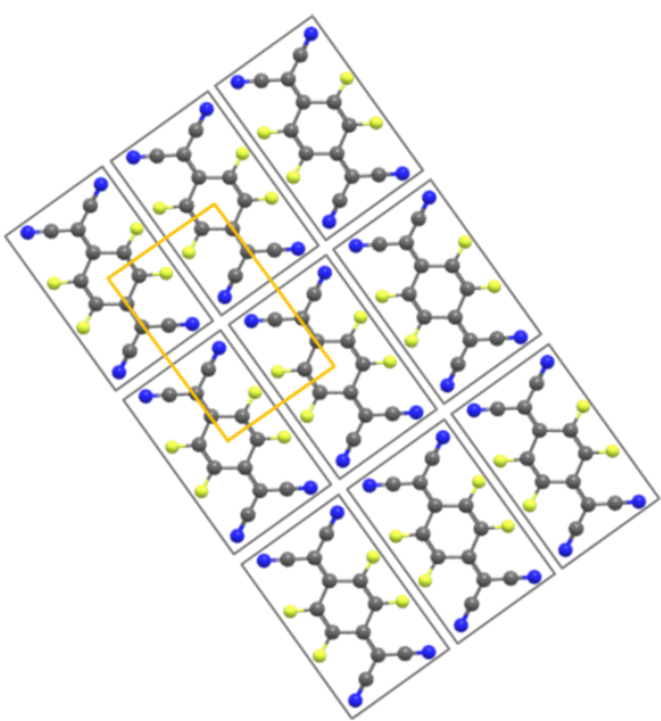

(b)

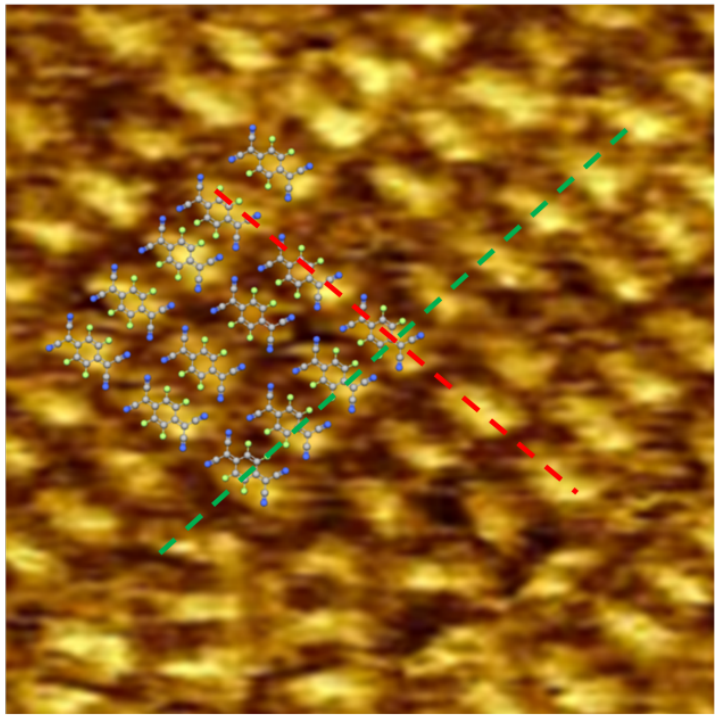

(d)
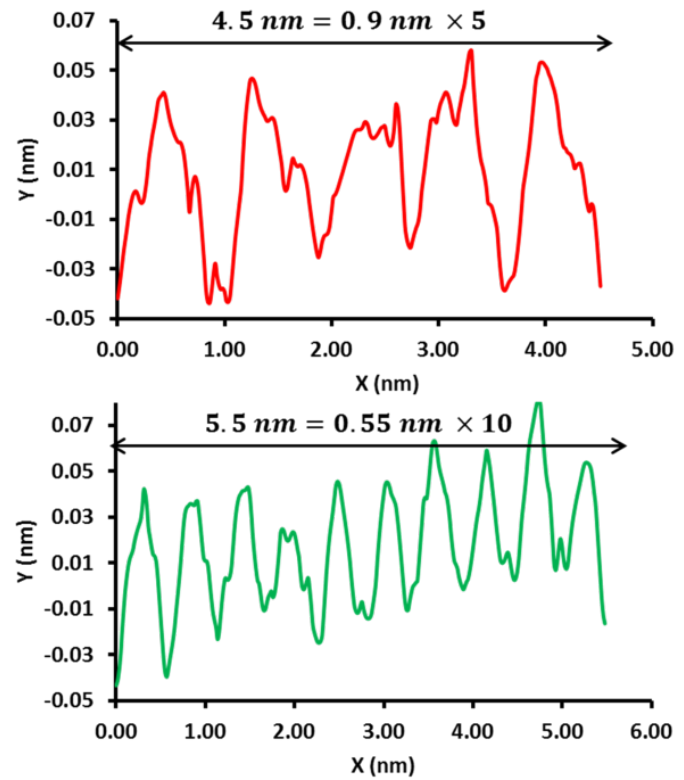

Figure S4. STM images of $\mathrm{F}_{4} \mathrm{TCNQ} / 0.05 \mathrm{M} \mathrm{H}_{2} \mathrm{SO}_{4}$ on $\mathrm{Au}(111)$ at $\mathrm{V}_{\mathrm{S}}=0.05 \mathrm{~V}_{\mathrm{SCE}}, \mathrm{V}_{\text {bias }}=0.03 \mathrm{~V}$ and $\mathrm{I}_{\mathrm{t}}=$ $0.1 \mathrm{nA}$ (a) scan area $50 \times 50 \mathrm{~nm}^{2}$, inset: $\mathrm{F}_{4} \mathrm{TCNQ}$ structure (b) Scan area $10 \times 10 \mathrm{~nm}^{2}$, (c) Proposed molecular packing and unit cell containing one $\mathrm{F}_{4} \mathrm{TCNQ}$ molecule, (d) Cross-sections of the ordered structure in the directions specified by the red and green lines in image (b) 


\section{d. STM images of $\mathrm{F}_{4} \mathrm{TCNQ}$ vs sample potential}

(a)

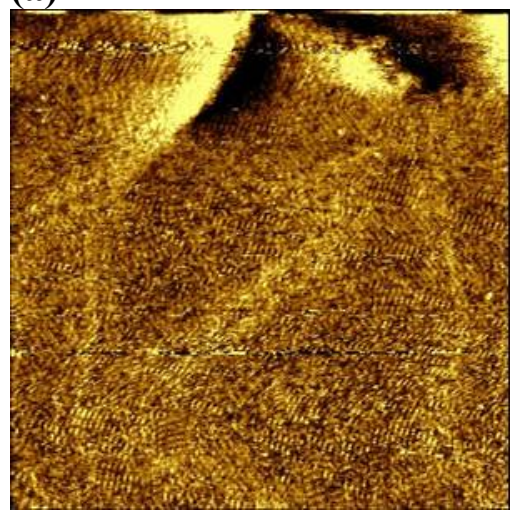

(d)

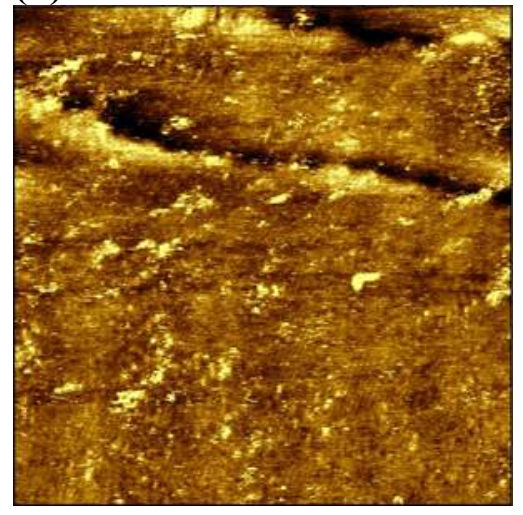

(b)

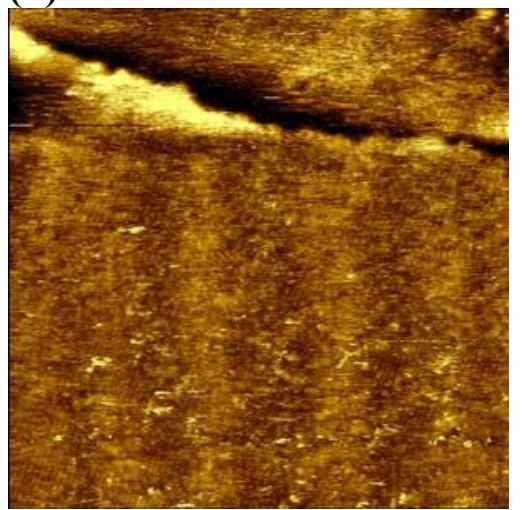

(e)

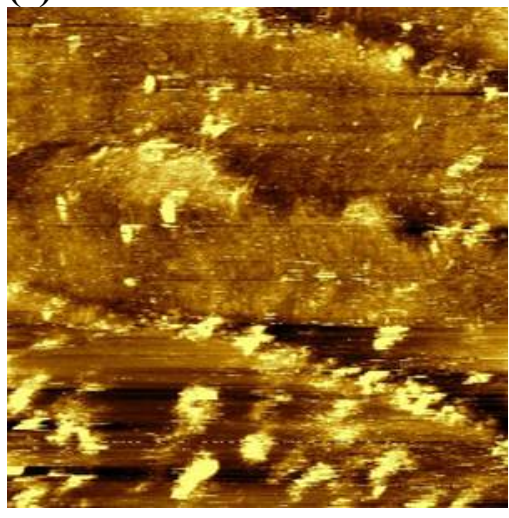

(c)

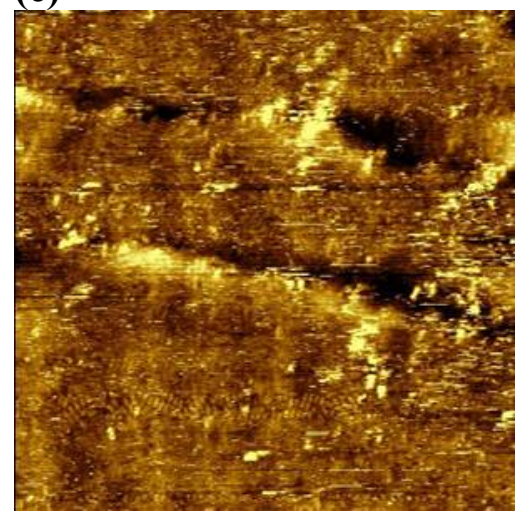

(f)

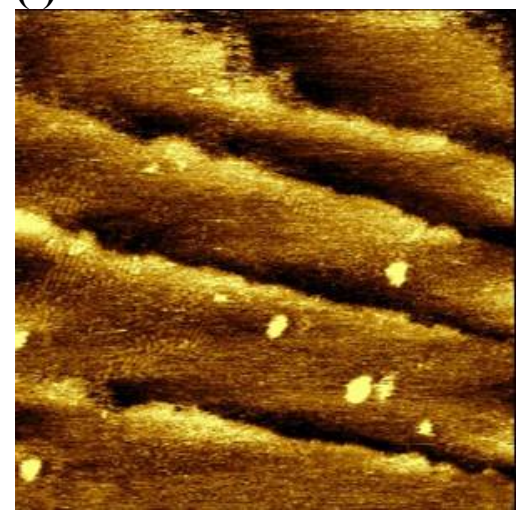

Figure S5. STM images of $\mathrm{F}_{4} \mathrm{TCNQ} / 0.05 \mathrm{M} \mathrm{H}_{2} \mathrm{SO}_{4}$ on $\mathrm{Au}(111), \mathrm{I}_{\mathrm{t}}=0.1 \mathrm{nA}$, scan area $50 \times 50 \mathrm{~nm}^{2}$ at (a) $\mathrm{V}_{\mathrm{S}}=0.05 \mathrm{~V}_{\mathrm{SCE}}$, (b) $\mathrm{V}_{\mathrm{S}}=0.20 \mathrm{~V}_{\mathrm{SCE}}$, (c) $\mathrm{V}_{\mathrm{S}}=0.35 \mathrm{~V}_{\mathrm{SCE}}$, (d) $\mathrm{V}_{\mathrm{S}}=0.50 \mathrm{~V}_{\mathrm{SCE}}$, (e) $\mathrm{V}_{\mathrm{S}}=0.70 \mathrm{~V}_{\mathrm{SCE}}$, (f) $\mathrm{V}_{\mathrm{S}}=0.10$ $\mathrm{V}_{\mathrm{SCE}}$

Consecutive STM images show the order/ disorder phase transition upon moving the sample potential to more positive values. The ordered structure disappeared completely at $\mathrm{V}_{\mathrm{S}}=0.35$ $\mathrm{V}_{\mathrm{SCE}}$ and started to reappear again at $\mathrm{V}_{\mathrm{S}}=0.10 \mathrm{~V}_{\mathrm{SCE}}$.

\section{4- In situ scanning tunneling microscopy-break junction (STM-BJ):}

\section{a. Method:}

Single molecule conductance measurements were performed using the scanning tunneling microscopy-break junction (STM-BJ) method with a PicoScan STM system (Molecular Imaging) in an electrochemical environment. 10 and $1000 \mathrm{nA} / \mathrm{V}$ gain current preamplifiers used for low and high SMC measurements, respectively.

In this method, the electrochemically etched gold sharp STM tip is brought into contact with the gold substrate while switching off the feedback loop. once the preamplifier reaches the maximum current, typically equivalent to the current associated with one or two Au-Au contacts, 
the tip is then retracted. As the tip withdraws, it can form an atomic Au-Au contact with a quantum of conductance $\left(G_{0}=\frac{2 e^{2}}{h}=7.75 \times 10^{-5} \mathrm{~S}\right)$. As the tip retracts it breaks the Au-Au contact and a nano gap can be formed between the tip and the gold surface. In presence of molecules, a single molecule can be wired in the nano gap and the electrical conductance of molecules can be measured. During the approaching and retracting, the process of the forming and breaking of junctions is repeated many times and a large number of current-distance traces are recorded, typically 2000-4000 traces in our experiments for statistical analysis.
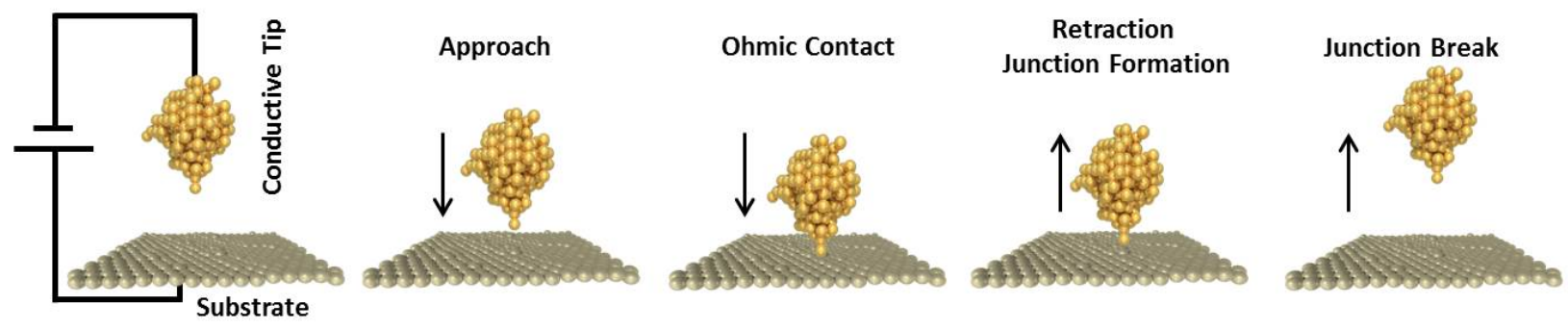

Figure S6. Principle of the scanning tunneling microscopy break-junction (STM-BJ) measurement

\section{b. Data selection criteria:}

To generate all data histograms in Figures 1, 2, and 3 in the manuscript and Figure S11-13 in the supporting information, we screened all of the recorded current-distance traces using a procedure, written in the Igor Pro software and that is available upon request, that rejects all traces that do not reach the saturation current $(10,000 \mathrm{nA}$ : the maximum current measured using the $1 \mu \mathrm{A} / \mathrm{V}$ preamplifier).

To have precise data selection in Figure 2 all current-distance curves were carefully checked one by one manually. Curves where no step is observed, curves with noise while the tip is contacting surface or breaking junction and non-monotonic curves are excluded by manual inspection.

We selected all curves with a step length greater than $0.1 \mathrm{~nm}$. Then, we distributed them to six different ranges based on the step current value and created a current histogram for each category. Some traces have more than one step, each belongs to different categories. These traces were saved based on their step current value in their assigned category.

Range 1: curves with step in range of $<5 \mathrm{nA}$

Range 2: curves with step in range of 5-10 nA

Range 3: curves with step in range of 10-20 nA

Range 4: curves with step in range of 20-30 nA

Range 5: curves with step in range of 30-50 nA

Range 6: curves with step in range of 50-90 nA 
A curve with two steps, for example at 15 and $55 \mathrm{nA}$ would be saved in the Range 2 and Range 5 data sets for subsequent analysis.

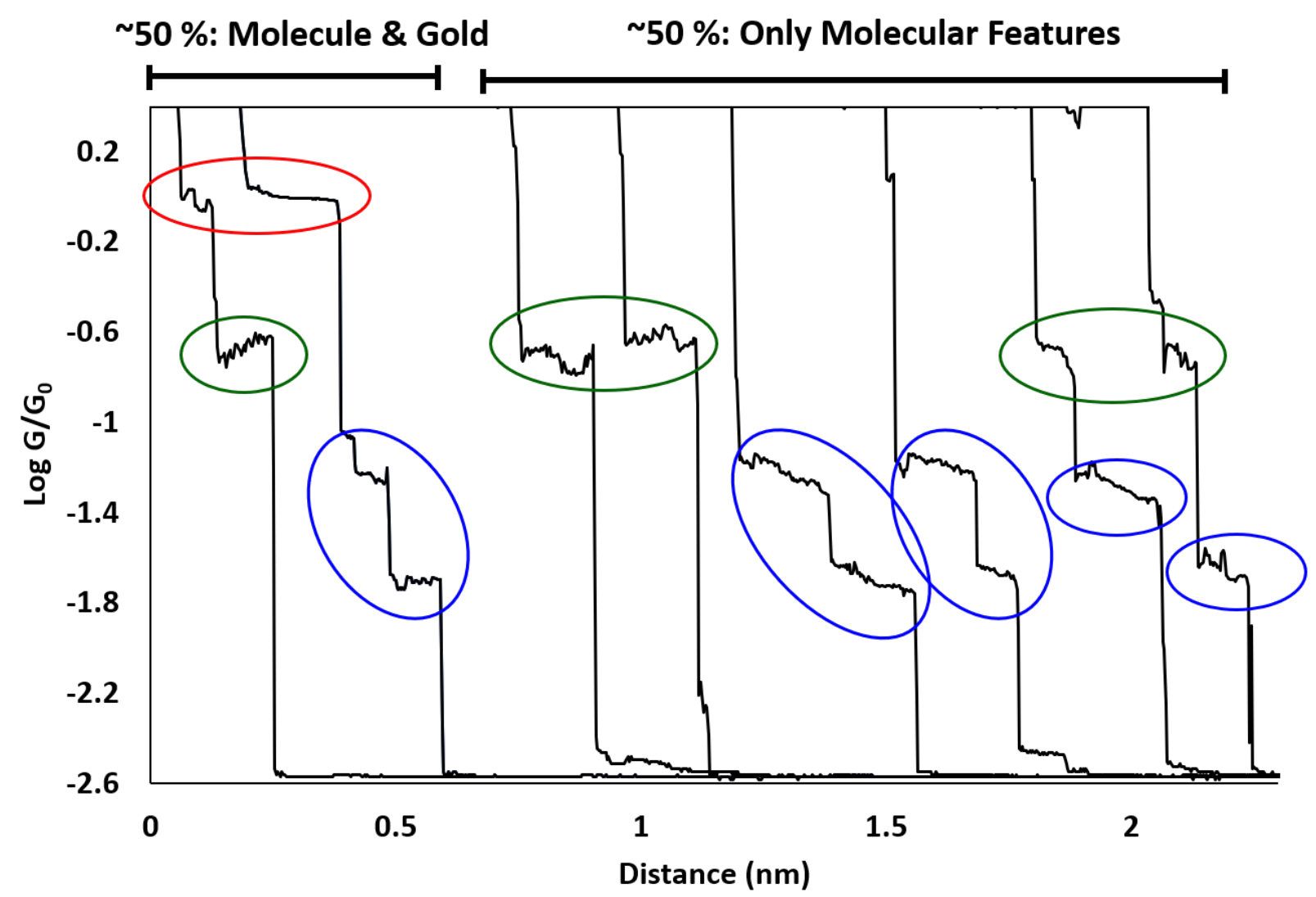

Figure S7. Examples of current-distance curves for $\mathrm{F}_{4} \mathrm{TCNQ} / 0.05 \mathrm{M} \mathrm{H}_{2} \mathrm{SO}_{4}$ on $\mathrm{Au}$ (111) at $\mathrm{V}_{\mathrm{S}}=0.05$ $\mathrm{V}_{\mathrm{SCE}}, \mathrm{V}_{\text {bias }}=0.05 \mathrm{~V}$. Red oval: Au-Au junction, Green oval: Flat molecular junction, Blue oval: $\mathrm{b}-\mathrm{b}$ and $\mathrm{m}-\mathrm{b}$ molecular junctions.

\section{MPVC analysis:}

\section{a. Description, method and data analysis:}

MPVC is essentially a dimensionality reduction technique that translates higher-dimensional current-distance (or similar) data into a low-dimensional classifier, while preserving as much relevant information as possible. Data traces that are similar to each other in the original data space are expected to be in close proximity in the lower dimensional MPVC representation as well. This means they can subsequently be clustered and separated, allowing for detailed analysis and quantification of potential sub-populations present in the original dataset. Specifically, all three classifiers used here, the vector distance $\Delta \mathrm{X}$, the cosine of the vector angle 
$\cos (\theta)$ and the reduced Hamming distance $\mathrm{d}_{\mathrm{ham}}$, are calculated relative to a common reference trace R:

$\Delta \mathrm{X}=\frac{\sqrt{\sum_{1}^{\mathrm{N}}\left(\mathrm{I}_{\mathrm{n}}-\mathrm{R}\right)^{2}}}{\mathrm{~N}}$

$\cos (\theta)=-\frac{\left(I_{n}-R\right) \cdot R}{\left|I_{n}-R\right| \cdot|R|}$

$d_{\text {ham }}=$ pairwise distance $\left(\operatorname{sign}(R), \operatorname{sign}\left(I_{n}-R\right),{ }^{\prime}\right.$ hamming $\left.{ }^{\prime}\right)(1 c)$

where the pseudo-code in eq. (1c) is a proxy for calculating the fraction of signs in the vector components that are different for the two vectors. The components of $\mathrm{R}$ do not change sign, but those of $\mathrm{I}_{\mathrm{n}}-\mathrm{R}$ do, because the current trace could be above or below $\mathrm{R}$.

In the present case, $\mathrm{R}$ was determined from the median of all data traces measured on the bare gold substrates and preserved for use with the TCNQ data. The underlying idea being that data traces without molecular events would be similar to the bare gold case, while those with specific 'molecular' information would form new clusters in the MPVC representation. While this is to some extent a simplification - the presence of the TCNQ could potentially change the transport characteristics of the junction, even if no molecule actually bridges the tunneling gap - the bare gold data still provide a reference frame for the analysis of the molecular data.

The result of this first step is shown in Figure S8 (a), with the data obtained from the bare gold substrate in red and the TCNQ data (low current region, measured with a $10 \mathrm{nA} / \mathrm{V}$ preamplifier) in black. In the former, two clusters are apparent, mainly separated along the $\cos (\theta)$ and $\mathrm{d}_{\text {ham }}$ dimensions, but at low $\triangle \mathrm{X}$. However, in the case of the TCNQ data, another pronounced point cloud extends to larger values of $\Delta \mathrm{X}$. We then performed k-medoid clustering with $\mathrm{N}=4$ clusters to capture the traces similar to those in the bare gold experiments (two clusters, as in the original bare gold data) as well as the regions between them and the one extending to larger $\Delta \mathrm{X}$ values. The result is shown in panel (b) with the groups color-coded and labeled accordingly, from 1-4 in blue, yellow, purple and green containing 3698, 1117, 2490 and 1268 data traces, respectively. While some cross-contamination between the clusters could not be avoided, the separation will be sufficient to enhance certain characteristics in the data that would otherwise go unnoticed, see below. For comparison and as a starting point, we show the 2D histogram of all 8573 data traces in panel (c). From this representation, it is clear that the picture is relatively complex, with some data traces decaying very quickly and others extending relatively far. 
(a)

(b)

(c)
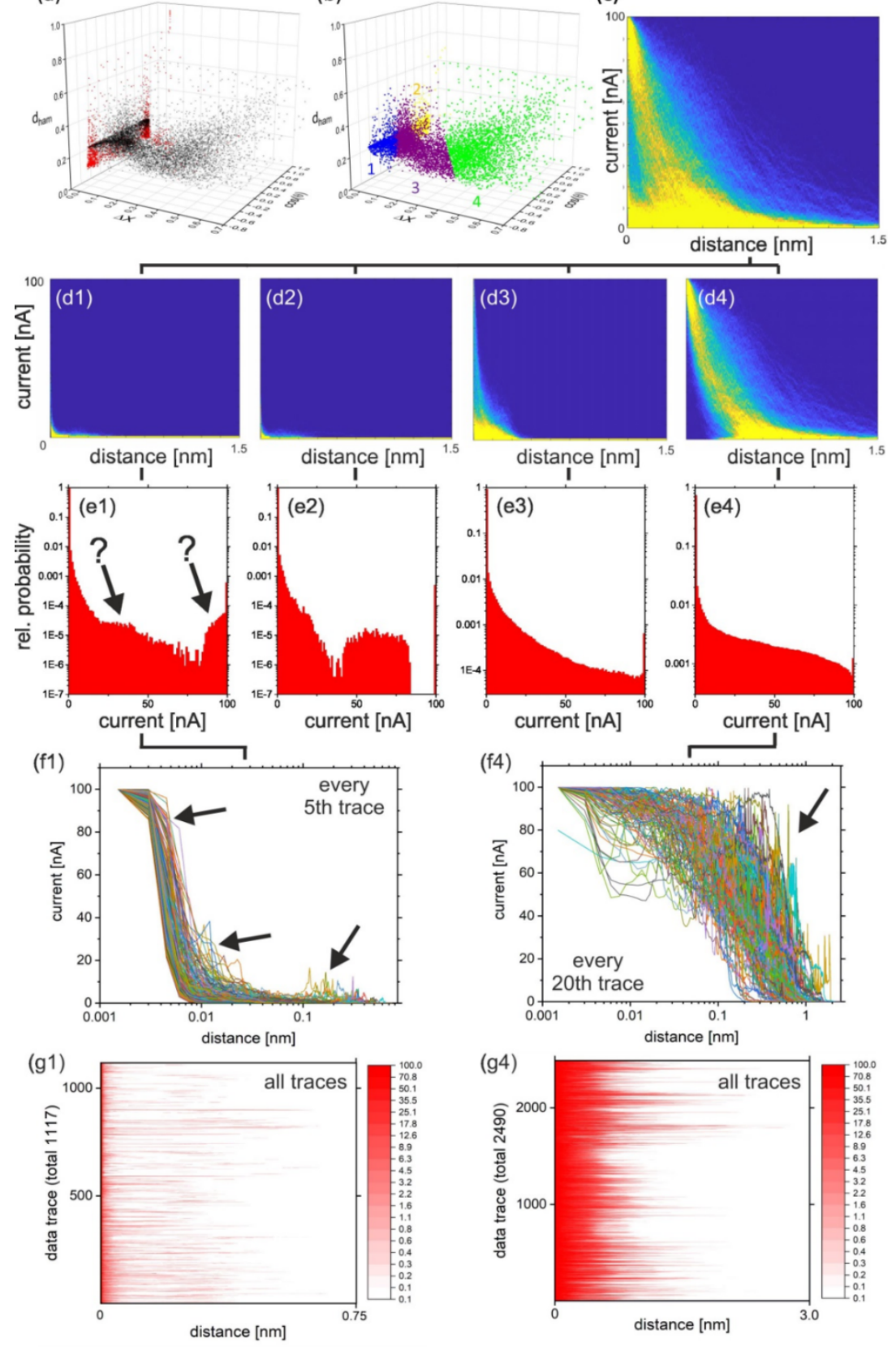

Figure S8. (a) 'All-data' cluster plots based on the three classifier components, $\Delta X, \cos (\theta)$ and $d_{\text {ham }}$, for the experiments on bare gold (red) and in the presence of TCNQ (black), cf. eq. (1a-c). (b) Clustering result of the TCNQ data (k-medoid based on 4 clusters), showing clusters 1 to 4 (blue, yellow, magenta and green, respectively). (c) All-data point 2D histogram of the TCNQ data. Different classes of the current-distance traces appear to be present. (d) $2 \mathrm{D}$ histograms of clusters 1 to 4 , (d1) to (d4). The separation of the different data classes is improved, even though the corresponding 1D histograms in panels (e1-4) do not feature clearly defined peak features (see main text for detailed discussion). Panels (f1) and (f4) show examples of individual current-distance traces, highlighting some of the common features within each cluster. This includes steep decays and noise features in $\mathrm{fl}$ and plateau-like features in (f4), which were also found in the manual selection based analysis. However, within this relatively large cluster (1268 out of 8573 traces), the variance is still large, and no single, well-defined conductance 
feature is observed. The heat maps in panels (g1) and (g4) show the large variation within clusters 1 and 4 , as well as the switching behaviour discussed in the main text.

The 2D histogram becomes somewhat clearer when broken down into the different clusters, as shown in the 2D histograms in panels (d1-4) (following the numbering of the clusters). Clusters 1 and 2 (d1) and (d2), encompass curves with rapid decays, which most likely correspond to 'molecule-free' electrode junctions, in line with the fact that very similar traces occurred in the 'bare gold' experiment (see panel (a)). Interestingly, these traces also feature a somewhat diffuse density region at low currents at distances between 0.15 and $0.3 \mathrm{~nm}$. We will investigate these for cluster 1, as an example below. Cluster 3 captures a mixture of rapidly decaying curves (d3), similar to those in clusters 1 and 2, and relatively well-defined, but less steep exponentially decaying traces. Further analysis showed that both sub-classes feature a region of high point density at low currents and distance, which, however, did not produce a characteristic signature (not shown). Finally, the traces in cluster 4 (d4) are relatively slowly decaying, albeit with considerable variance within the group. There does not appear to be any well-defined molecular feature in cluster 4. Since the conductance of some TCNQ configurations is rather high (and outside the corresponding current range for the low-conductance data, $100 \mathrm{nA}$ ), it is possible that some electrode-molecule interaction is taking place, albeit without a well-defined break-off point. The one-dimensional histograms (Figure S8, e1-4), however, highlight features that were also recovered in the manually-selected data, i.e., molecular features close to the limit of the lowconductance range (just below $100 \mathrm{nA}\left(0.026 \mathrm{G}_{0}\right)$ ) and at around $\sim 30 \mathrm{nA}\left(0.008 \mathrm{G}_{0}\right)$. Some of these are highlighted with the arrows in panel (e1) (cluster 1), for example. Indeed, further inspection of the original data traces in panel (fl) (every 5th trace plotted) reveals that there is some higher abundance of current values close to $80-90 \mathrm{nA}$, albeit no well-defined molecular characteristic, and some plateau-like features around 30-40 nA. The latter, however, do not seem to form a well-defined conductance based on the MPVC analysis, which is also why this characteristic does not feature in the $2 \mathrm{D}$ histograms. In addition, the traces shown in (f1) also feature some characteristic noise patterns at distances from 0.1 to $0.2 \mathrm{~nm}$. We found that this sort of switching behaviour was rather common, even though its stochastic nature prevents a strong presence in the 2D histograms. As an alternative, we show a heat map in panel ( $\mathrm{g} 1)$, which illustrates this behaviour somewhat better. Similar switching behaviour was also found in cluster 2, which is responsible for the feature in the 1D histogram (e2), between current values of approximately 40-80 nA (0.01-0.02 $\left.\mathrm{G}_{0}\right)$. 1D histograms (e3) and (e4) are rather featureless, even though individual data traces show switching and a range of different plateau features between approximately 50 and $100 \mathrm{nA}$, again without forming a distinct group of events. For cluster 4, this is again illustrated in panels (f4) (every 20th trace plotted) and (g4) (heat map).

Thus, while the conclusions from the analysis-based manually-selected data could not be fully confirmed by MPVC, for example in terms of specific conductance families, the main features are reproduced. Namely, MPVC shows the emergence of molecular features when the target molecules are present, in comparison to experiments with unmodified, bare Au substrates. With a view to the molecular interpretation of the data, MPVC results seem to be in line with the 
notion that junctions are relatively poorly defined in this conductance region, perhaps involving varying molecular configurations during the process.

\section{b. Comparison of manual selection vs MPVC classification of low-conductance data}

In relation to the different data selection procedures employed in this work, namely manual selection vs. MPVC-based selection, we provide here a more detailed, direct comparison of the two approaches. As mentioned, the selection criteria are not identical; hence one would not expect a one-to-one correspondence. Nevertheless, some relation between the cluster allocation and the hand-selected classification should be apparent and this is indeed observed, as shown below.
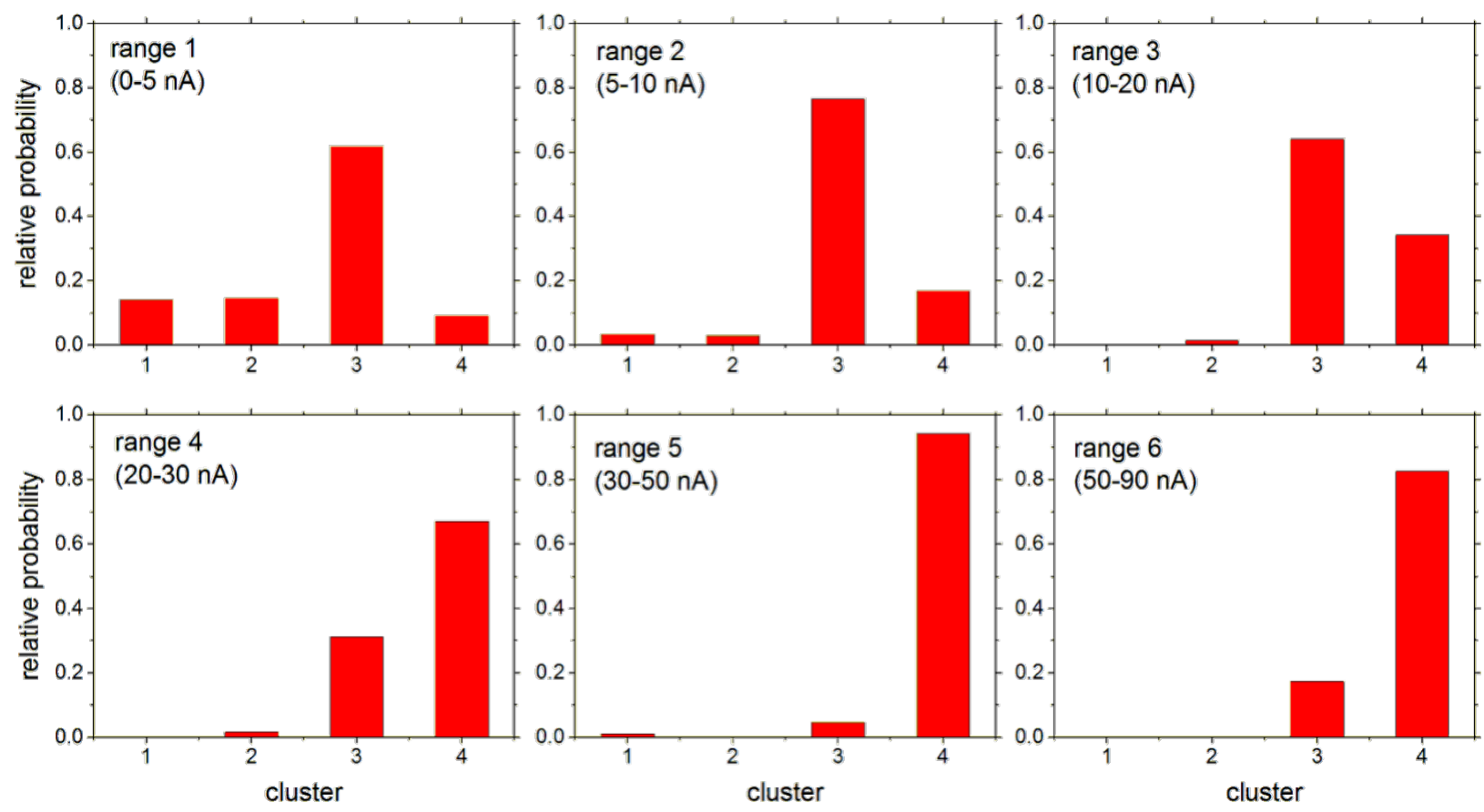

Figure S9: Allocation of manually-selected data to the four MPVC clusters, for the six ranges stated above (normalized). Manually-selected traces are predominantly found in clusters 3 and 4, where the former dominates at low-current features (especially ranges $1+2$ ), while the latter mainly encompasses traces with high-current features (mainly ranges $5+6$ ). 

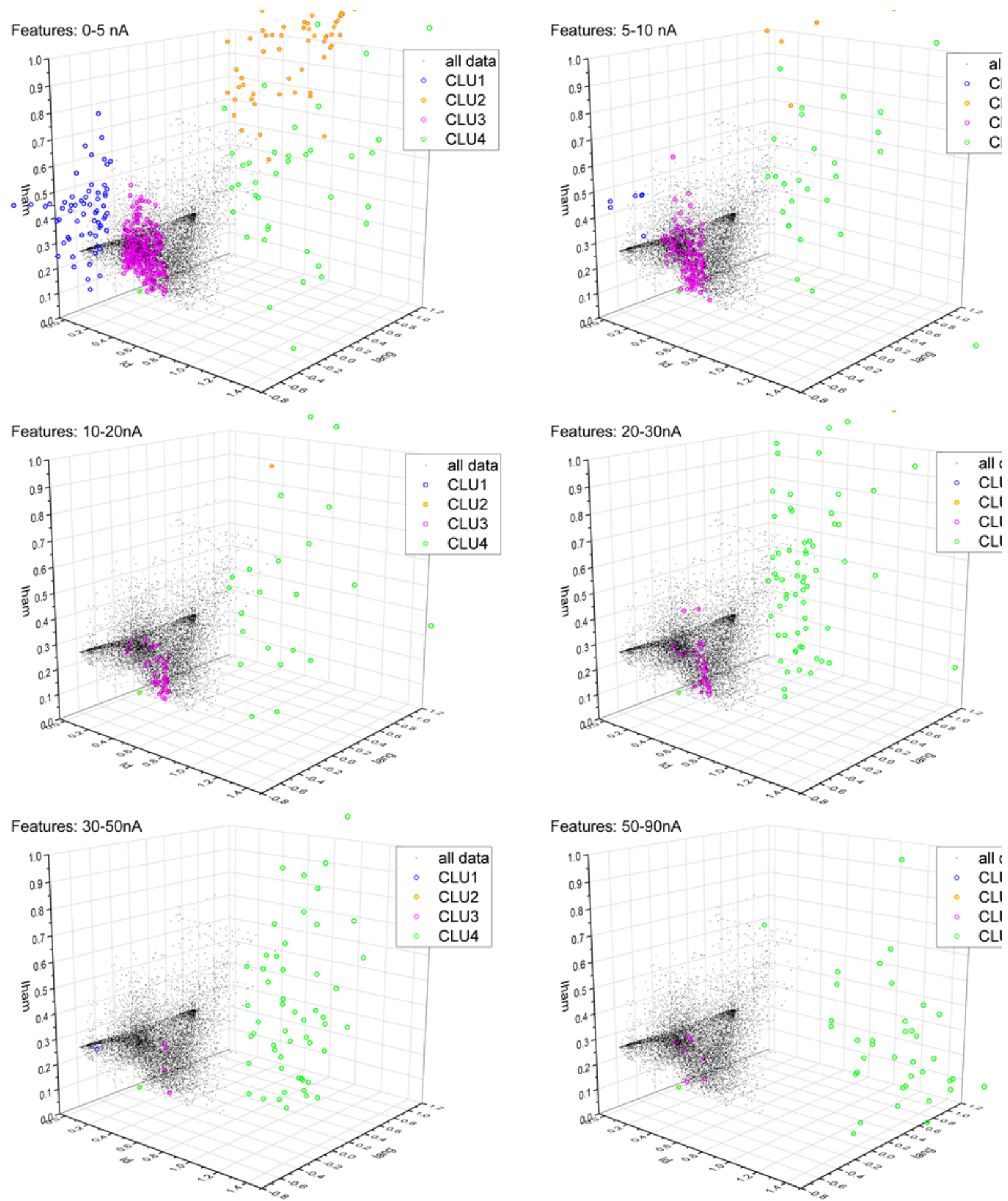

Figure S10: Cluster plot representation with data from manual selection in the different ranges colorcoded according to cluster assignment. 


\section{6- STM-BJ control experiments:}

(a)

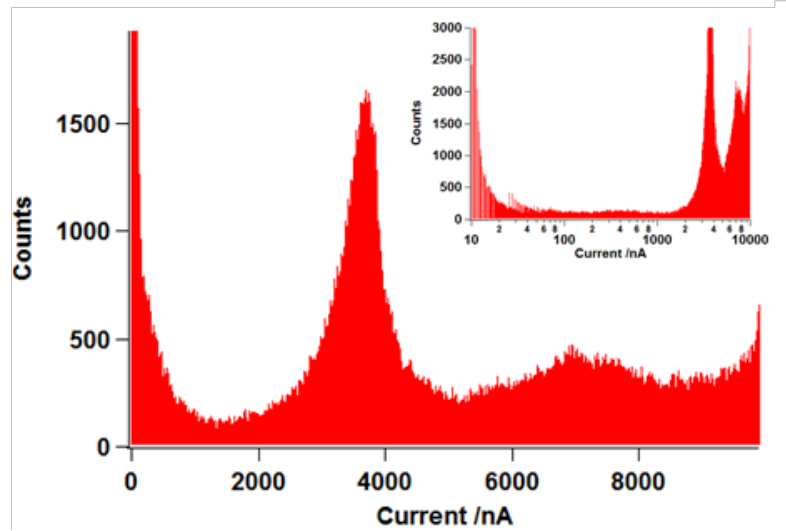

(b)

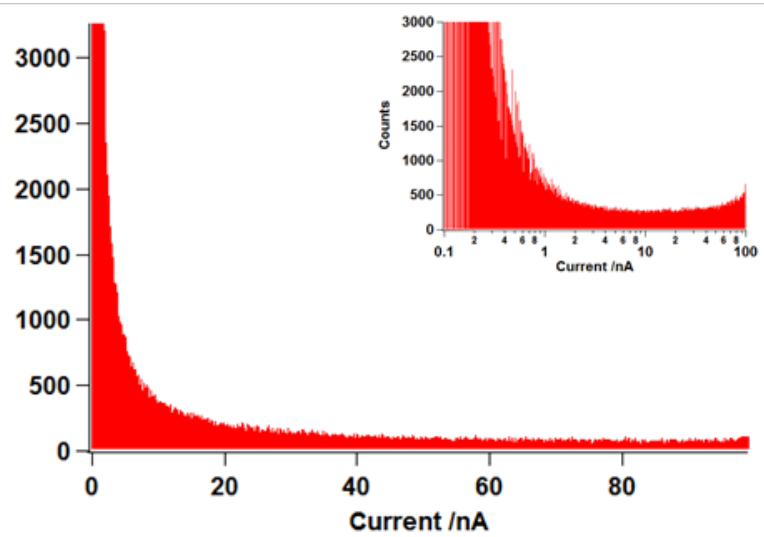

Figure S11. STM-BJ control experiment in $0.05 \mathrm{M} \mathrm{H}_{2} \mathrm{SO}_{4}$ on (a) negatively charged $\mathrm{Au}(111), \mathrm{V}_{\mathrm{S}}=0$ $\mathrm{V}_{\mathrm{SCE}}, 2356$ curves and (b) $\mathrm{V}_{\mathrm{S}}=0.70 \mathrm{~V}_{\mathrm{SCE}}, 3213$ curves. Insets are log-binned histograms constructed with the same curves used for 1D current histograms.

\section{7- Single molecule conductance vs sample bias:}

To substantiate that the observed SMC peaks are a signature of single TCNQ and $\mathrm{F}_{4} \mathrm{TCNQ}$ molecules, SMC experiments were carried out with different biases. As we can see in Figures S12 and S13, the current maximum increases linearly with bias, indicating the bias independent molecular conductance.

(a)

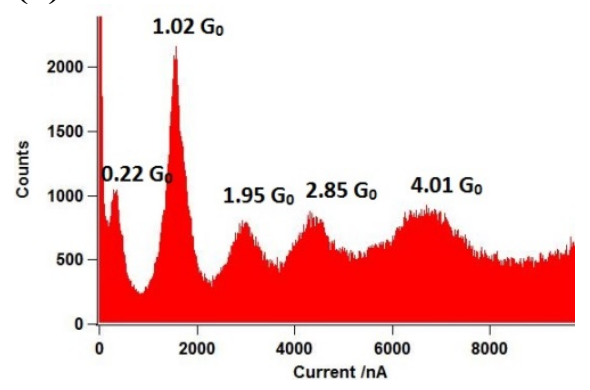

(b)

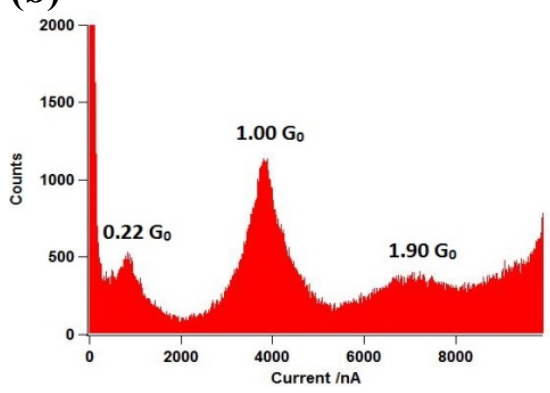

(c)

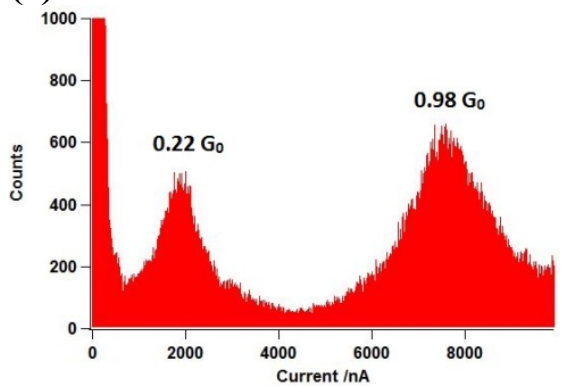

Figure S12. STM-BJ experiments of TCNQ carried out at different biases: All data (a) $\mathrm{V}_{\text {bias }}=0.02 \mathrm{~V}, 2391$ curves (b) $\mathrm{V}_{\text {bias }}=0.05 \mathrm{~V}, 3176$ curves (c) $\mathrm{V}_{\text {bias }}=0.10 \mathrm{~V}, 3226$ curves

(a)

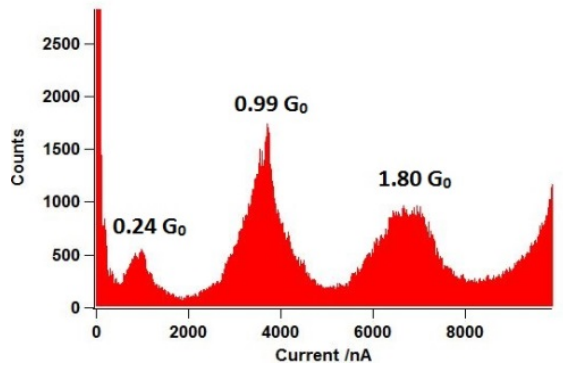

(b)

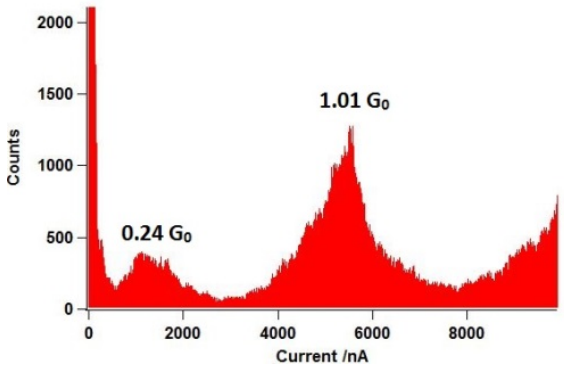

(c)

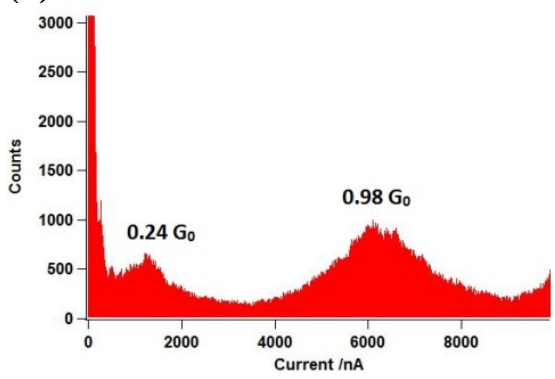

Figure S13. STM-BJ experiments of $\mathrm{F}_{4} \mathrm{TCNQ}$ carried out at different biases: All data at (a) $\mathrm{V}_{\text {bias }}=0.05 \mathrm{~V}$, 
3563 curve (b) $\mathrm{V}_{\text {bias }}=0.07 \mathrm{~V}, 2108$ curves (c) $\mathrm{V}_{\text {bias }}=0.08 \mathrm{~V}, 2749$ curves

\section{8- DFT Calculations:}

\section{a. Method:}

Ground state, $\Gamma$-point, density functional theory (DFT) calculations were performed on the molecule-electrode system with the Vienna Ab-Initio Simulation Package (VASP) $)^{5}$ using the $\mathrm{PBE}^{6}$ functional as the exchange-correlation functional. The method of Grimme ${ }^{7}$ (D3) was used to account for van der Waals (vdW) interactions. The Kohn-Sham single particle wavefunctions were represented by a plane wave basis set for valence electrons with a kinetic energy cutoff of $400 \mathrm{eV}$ along with projector augmented wave $(\mathrm{PAW})^{8}$ potentials to capture ion-core electron interactions. Ion positions were optimized using a conjugate gradient algorithm ${ }^{5}$ until net forces on ions were below $0.01 \mathrm{eV} / \AA$.

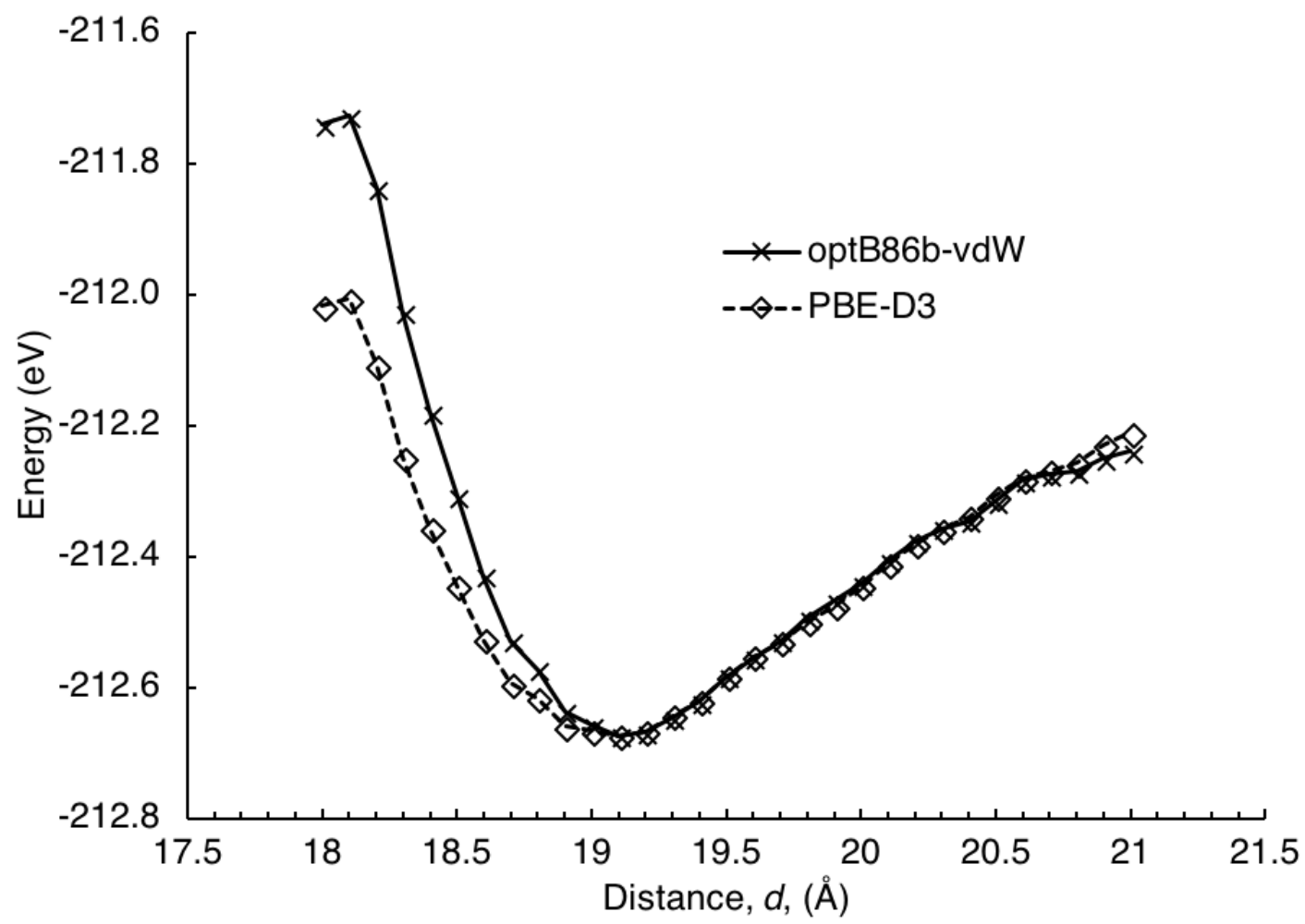

Figure S14. Comparison of electrode-molecule system (flat geometry) energy at varying electrode distance, $d$, found using the optB86b-vdW and PBE-D3 functionals. The minimum energies coincide at a distance of $19.1 \AA$. Energies points found with PBE-D3 are shifted by a constant equal to the difference between the energy values found at $19.1 \AA$ by the two functionals, such that the points at $19.1 \AA$ coincide on the plot.

The D3 correction is not as sophisticated as current van der Waals density functionals (vdW-DF) but was used in the interest of computational efficiency. To give confidence to our choice of 
$\mathrm{vdW}$ correction, energy calculations were repeated for several proposed geometries using the optB86b-vdW ${ }^{9}$ functional yielding the same minimum electrode separations as PBE-D3 and minimal change in the electrode-molecule geometry. The energy as a function of electrode separation for the flat $\mathrm{F}_{4} \mathrm{TCNQ}$ system is plotted in Figure S14 for both functionals. The plots only disagree qualitatively once the electrode separation moves well below the minimum energy separation; therefore, we affirm that the minimum energy separation and the geometry of the electrode-molecule system at the minimum are in good agreement.

To perform transport calculations 'buffer layers' are added to the optimized geometry. Figure S15a shows the system in which minimum energy calculations were performed while Figure $\mathrm{S} 15 \mathrm{~b}$ shows the corresponding scattering region used in the transport calculations. To calculate the transmission in Nanodcal ${ }^{10-11}$, the non-equilibrium electron density is determined for the system in Figure S15b, using the PBE functional, followed by a calculation of the transmission coefficients.

a)

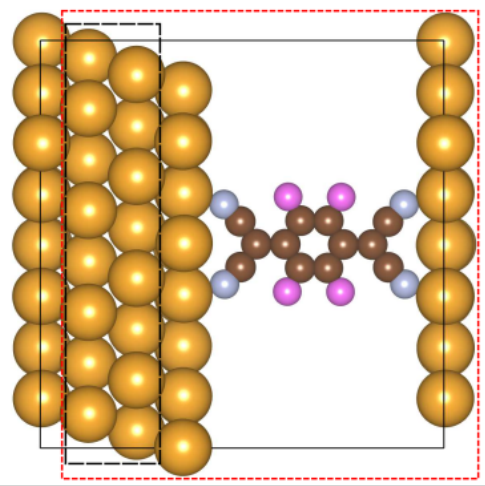

b)

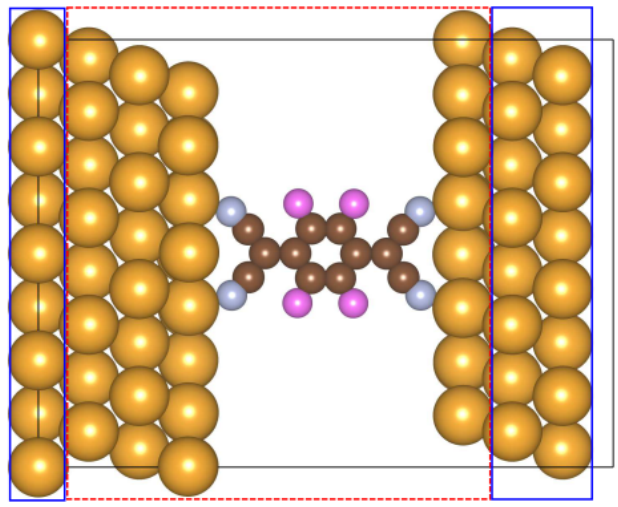

Figure S15. a) Unit cell (solid black) of the bidentate-bidentate system used in geometry optimization, showing fixed Au layers in the black dashed box. The dotted red box shows the region which is left unchanged when making the b) scattering region for transport calculations. Additional buffer layers (solid blue boxes) are added in constructing the scattering region.

\section{b. $F_{4}$ TCNQ - Geometries and Transmission}

To get an idea of how the geometry of the electrodes (e.g. shape, number of layers) affect the conductance, calculations were repeated with several different electrode models. In general, the low-bias conductance did not vary significantly. The bidentate-bidentate (b-b) geometry was modeled with four different electrodes (counting the $b-b$ model used in the main text). One is shown in the main text (Figure 4) and the other three are shown in Figure S16. Flat electrodes were used again, only this time with two layers free to move (Figure S16a) and flat electrodes with three layers free to move (Figure S16b). In the third model (Figure S16c), two Au atoms are placed on each electrode such that each $\mathrm{N}$ atom can bind to it. The same procedure outlined in the main text is used to calculate the low-bias conductance of these models. 
a)

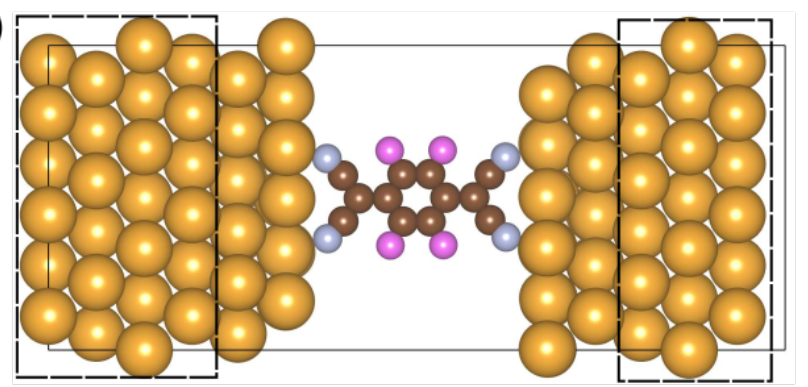

b)

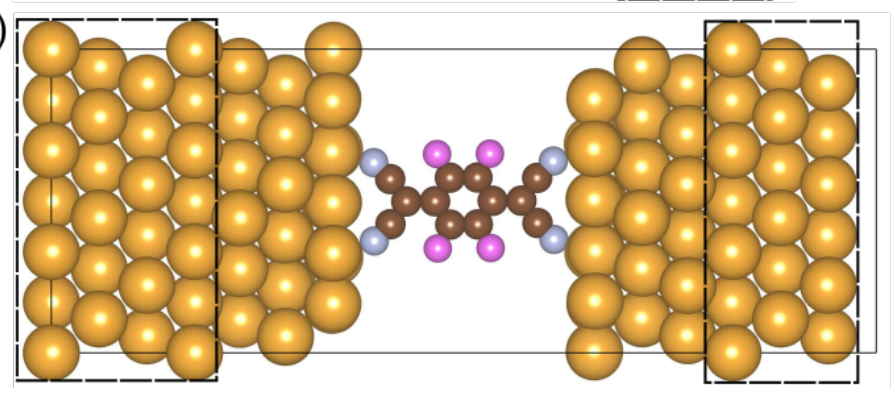

c)

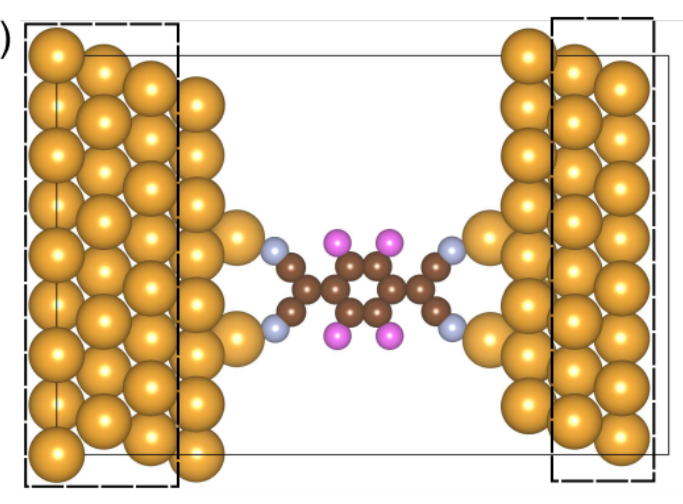

Figure S16. Alternate bidentate-bidentate system unit cells (solid black) in which transmission calculations were performed: a) flat electrodes with two Au layers free to move, b) flat electrodes with three $\mathrm{Au}$ layers free to move, and c) one Au layer free to move (same as in main text) only with two extra $\mathrm{Au}$ atoms added to each electrode surface. The dotted black box shows the fixed layers during optimization.

For the sake of computational efficiency, the number of Au atoms per layer was reduced when adding more layers to the system (Figure S17a and b). Due to the orientation of the molecule, this reduction in cross-section still leaves ample room $(10 \AA)$ between each molecule to avoid interaction between molecule images. When comparing the conductance values, it should be noted that the decrease in cross section (Figure S17a and b) was not accompanied by an increase in the $k$-point mesh while performing geometry optimizations. In other words, one should assume that the system in Figure S16b is an improvement of the flat electrode model in (a) and that only (c) is on the same footing for comparison as the bi-bi model in the main text but is a different electrode model, i.e. not flat electrodes. The low-bias conductances of (a), (b), and (c) were found to be $0.03,0.02$, and $0.01 \mathrm{G}_{0}$, respectively. Recalling that the computed value of the b-b system in the main text was $0.02 \mathrm{G}_{0}$, the low-bias conductance is relatively consistent (order of magnitude) among these four different models. This suggests that the choice of model electrode does not have a large influence on the overall conductance for a given configuration. 

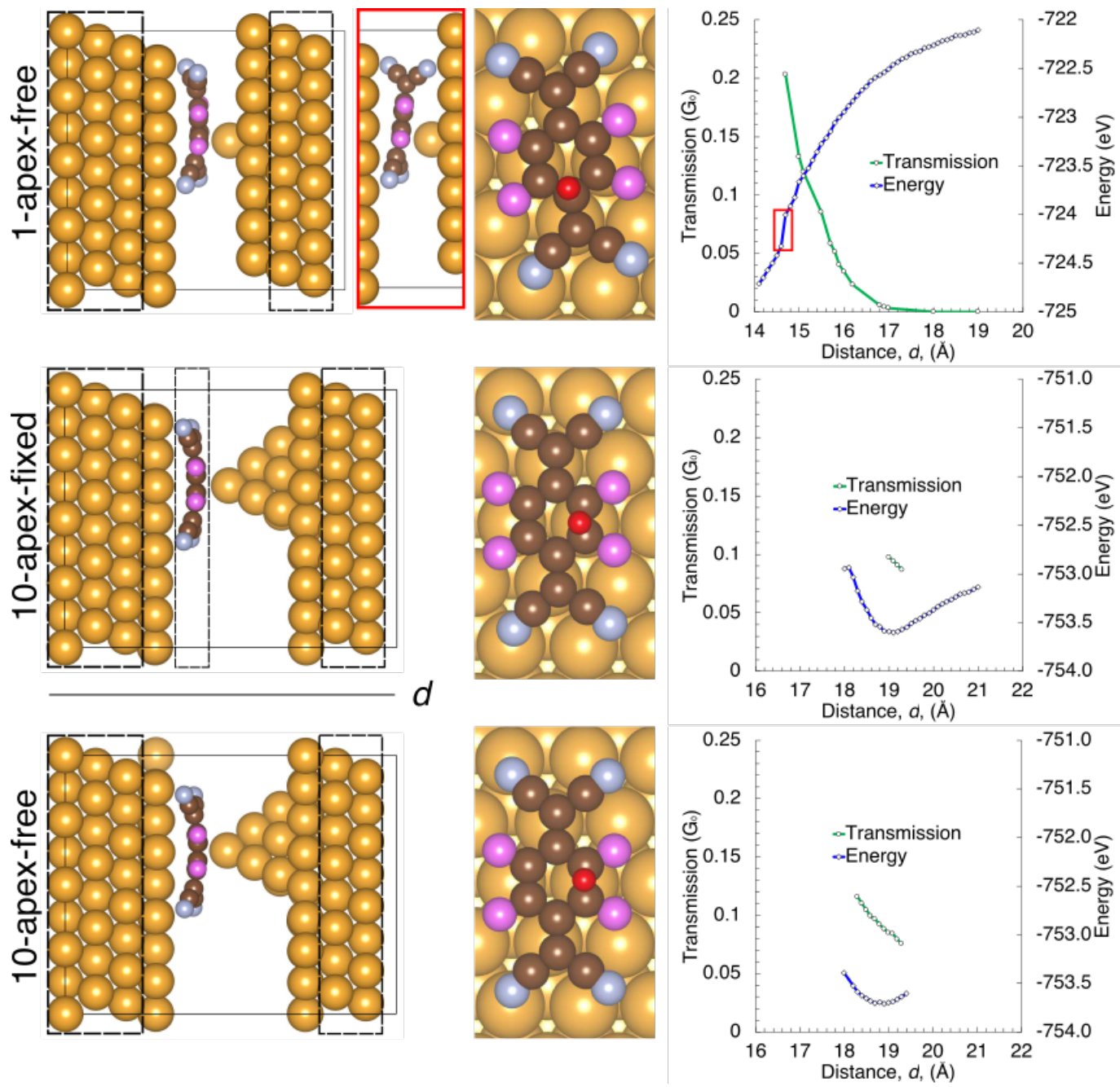

Figure S17. From left to right: side and top views of the lowest energy geometries, and transmission (at the Fermi level) and energy plots of three different methods used in calculating the low-bias conductance for the flat geometry. In the top view, the small red atom illustrates the alignment of the Au tip above the molecule.

Different models for the flat configuration are shown in Figure S17. The 'free' or 'fixed' in each configuration name refers to the molecule itself. In all models, the molecule is first relaxed on the Au surface prior to the apex (electrode on the right) being brought into close proximity of the molecule. In the 10-apex-fixed model, the molecule was then constrained to that position during the changing of the electrode distance, $d$. Both 10-apex models gave a low-bias conductance of $0.1 \mathrm{G}_{0}$ at their energy minima. In the 1-apex-free model, a minimum energy distance was not determined because the molecule attached, via a $-\mathrm{CN}$ anchor, to the apex electrode (image in red box of Figure S17) prior to finding the optimal coupling between the single Au apex and carbon atom. Just before attachment $(d=14.7 \AA$ on the plot), for which top and side views are shown, the low-bias conductance is $0.2 \mathrm{G}_{0}$. The factor of two discrepancies between the 10-apex and 1apex models is suspected to come from the two different alignments of the Au apex above the molecule, whose frontier orbital has nodal regions throughout. 
The apex-molecule alignment is somewhat discretized by the $\mathrm{Au}(111)$ stacking due to the reliance of our model electrodes on periodic boundaries. A model which includes vacuum between electrodes would allow a continuous variation in alignment. Such a model could better explore the dependence of tip alignment on the conductance, but is beyond the scope of this work.
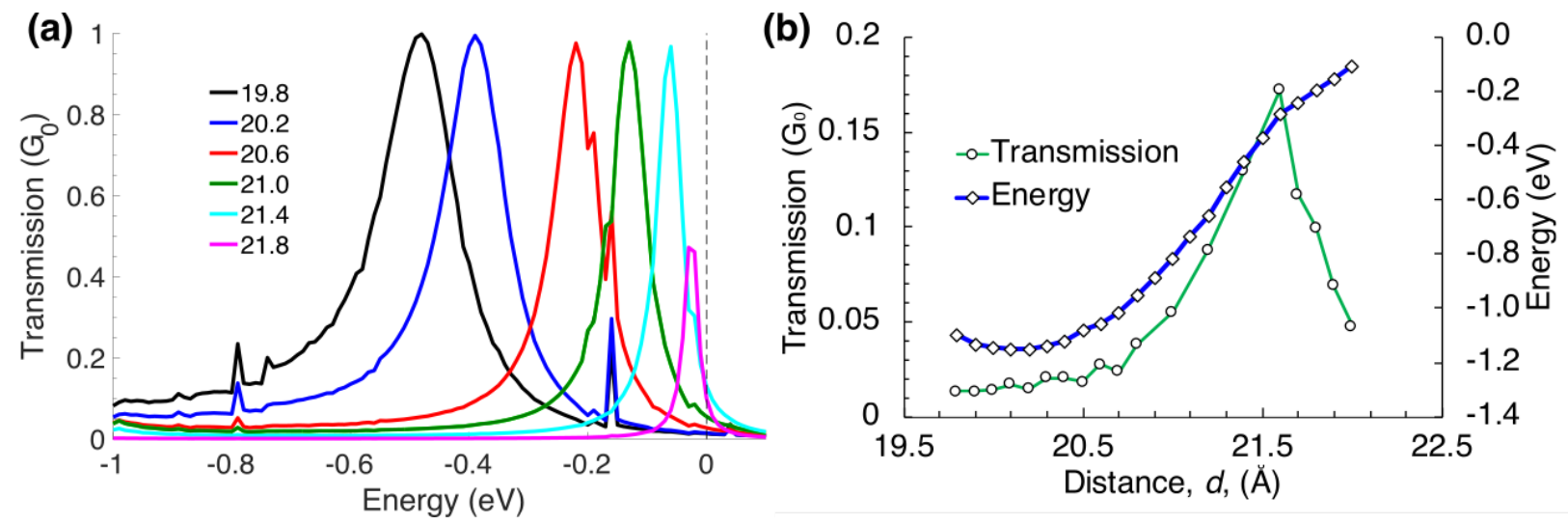

Figure S18. a) Calculated transmission spectra of bidentate-bidentate anchored $\mathrm{F}_{4} \mathrm{TCNQ}$ for various electrode distances and b) the energy and low-bias transmission plotted as a function of electrode distance.

Transmission spectra are calculated for different electrode distances. Several transmission spectra for the bidentate-bidentate system are shown in Figure S18a. As the electrode distance increases, the peak becomes narrower suggesting that the electrode-molecule coupling is diminishing. As the $\mathrm{F}_{4} \mathrm{TCNQ}$ molecule decouples from the gold electrodes, the peak (associated with the LUMO) lying below the Fermi level moves into better alignment with the Fermi level, increasing the conductance. This is seen more clearly in Figure S18b, which shows the low-bias conductance as a function of electrode distance. This effect, known as force-induced resonant tunneling ${ }^{12-13}$, predominantly occurred in the $b-b$ and $m-b$ calculations. The effect has been observed in previous experiments with benzene dithiol ${ }^{14}$. One would expect experimental factors such as thermal effects or molecular collisions (which are not captured by these calculations) to prevent such a maximally decoupled orientation from remaining in that state.

\section{c. TCNQ - Geometries and Transmission}

The results for TCNQ share many similarities with F4TCNQ. The same effect previously illustrated in Figure S18 (increasing conductance in the b-b system as the junctions are stretched) can be seen in the transmission plot in Figure S19 for b-b TCNQ. This effect is more exaggerated compared to $\mathrm{F}_{4} \mathrm{TCNQ}$. 

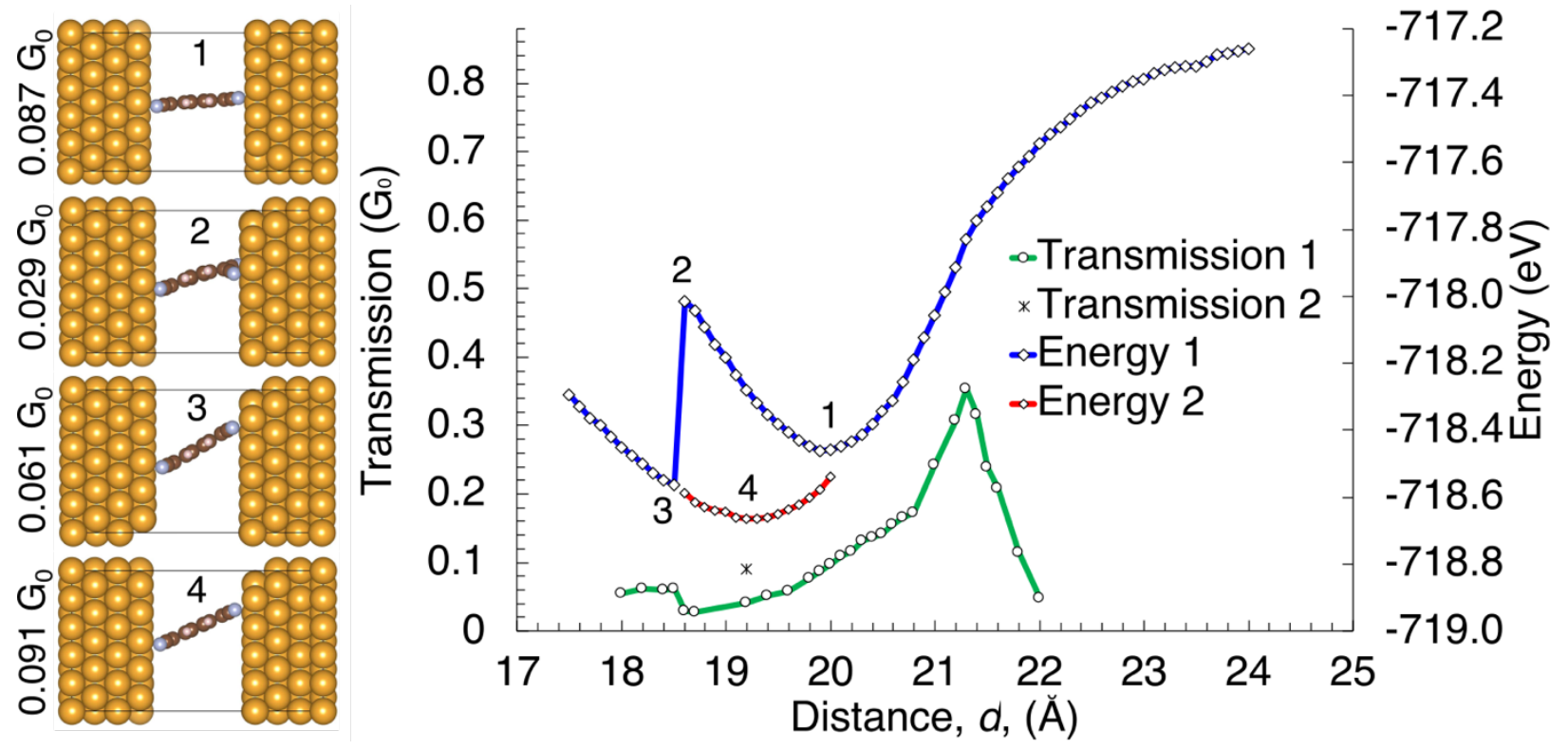

Figure S19. Side views and plots of energy and low-bias transmission as a function of electrode distance, $d$, of the bidentate-bidentate geometry with flat Au electrodes. Two minima are found by shortening and stretching the junction.

Several stretching and shortening cycles were performed in the b-b system. Geometries of interest during the progression are shown in Figure S19 and labeled 1, 2, 3, and 4. The b-b electrode separation is initiated at $19.8 \AA$ and stretched through the equilibrium point, 1 . The electrodes are also shortened from $19.8 \AA$ through 2 and 3. Then the electrodes are stretched again, starting from 3 , through the new minimum at 4 . In the b-b geometry at 1 , the left and right anchoring groups are attached to two pairs of $\mathrm{Au}$ atoms aligned directly across from each other in the junction. During the shortening process a first $-\mathrm{CN}$ anchor shifts to the adjacent Au atom (2), possibly distorting the extended $\pi$-system of the molecule. At 3, the second - $\mathrm{CN}$ anchor, on the same electrode, follows the first and shifts to the adjacent $\mathrm{Au}$ atom, allowing the molecule to straighten out again. The geometries at 1 and 4 are both minima because they are the optimal electrode-molecule coupling distance, but for slightly different $b-b$ junctions. There is an increase in conductance of $0.03 \mathrm{G}_{0}$ to $0.06 \mathrm{G}_{0}$ moving from the distorted geometry at 2 to the straightened geometry 3 . This suggests the conductance is fairly sensitive to the alignment of the extended $\pi$-system. The conductances at the two minima, 1 and 4 , are very similar at $0.087 \mathrm{G}_{0}$ and $0.091 \mathrm{G}_{0}$, respectively. This illustrates the validity of using the Au-N coupling as a defining factor in the overall conductance for a given denticity, e.g. b-b. 

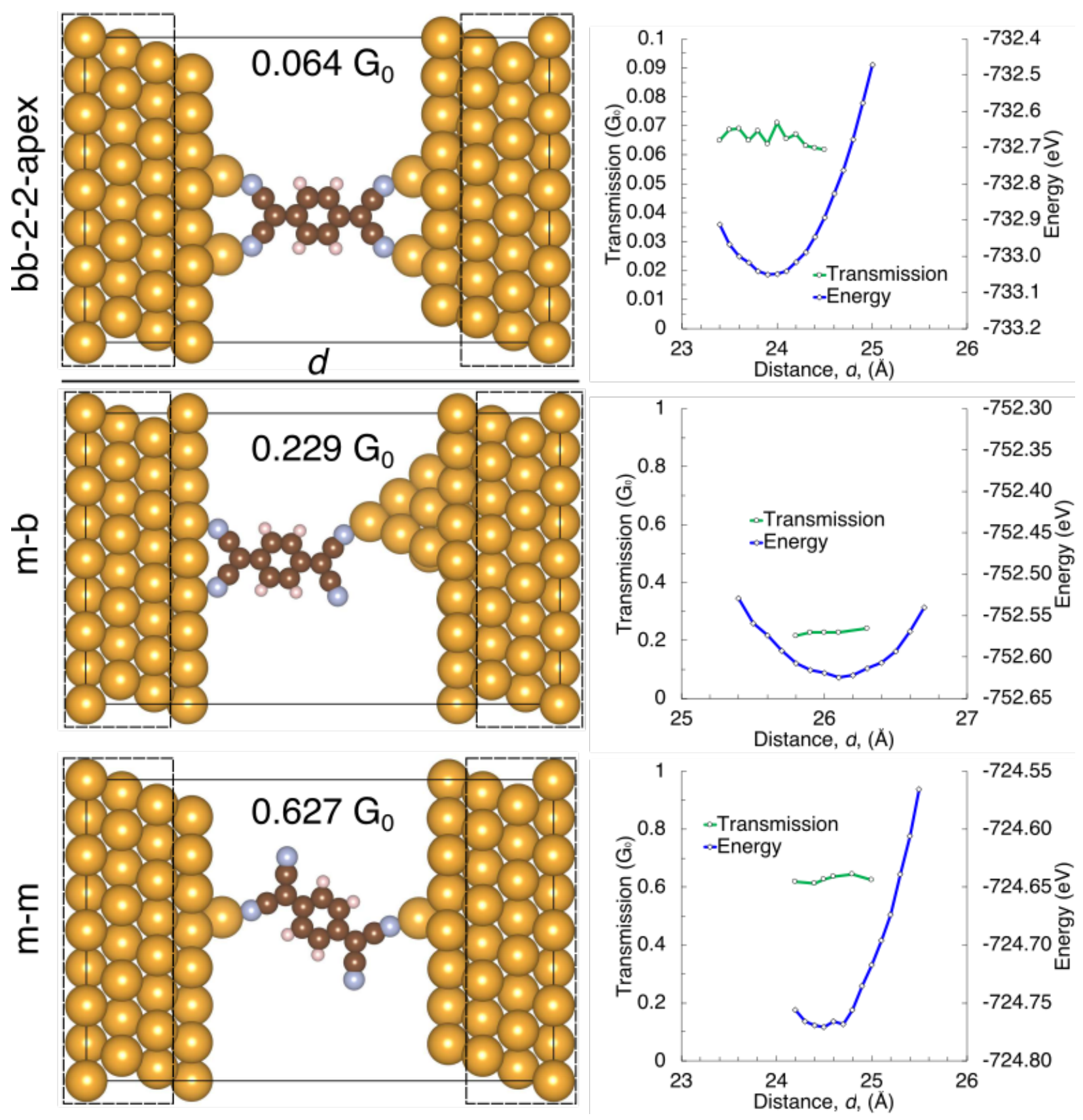

Figure S20. Side views and plots of energy and low-bias transmission as a function of electrode distance, $d$, of three different TCNQ configurations. The geometries and transmissions shown correspond to the energy minima. Black dotted lines enclose those atoms fixed during optimization.

The b-b system with two single Au atom apexes on both electrodes (bb-2-2-apex in Figure S20) gives a conductance of $0.064 \mathrm{G}_{0}$, somewhat lower than that of the flat $b-b$ electrode system. A similar trend was found with $\mathrm{F}_{4} \mathrm{TCNQ}$ whose conductance decreases by $\sim 0.01 \mathrm{G}_{0}$ while with TCNQ the conductance decreases by $\sim 0.03 \mathrm{G}_{0}$. The transmission as a function of $d$, shown in Figure S20, stays fairly constant at least for the range of $d$ calculated. The $\mathrm{m}-\mathrm{b}$ and $\mathrm{m}-\mathrm{m}$ conductance remains consistent at $\sim 0.2 \mathrm{G}_{0}$ and $\sim 0.6 \mathrm{G}_{0}$, respectively. 

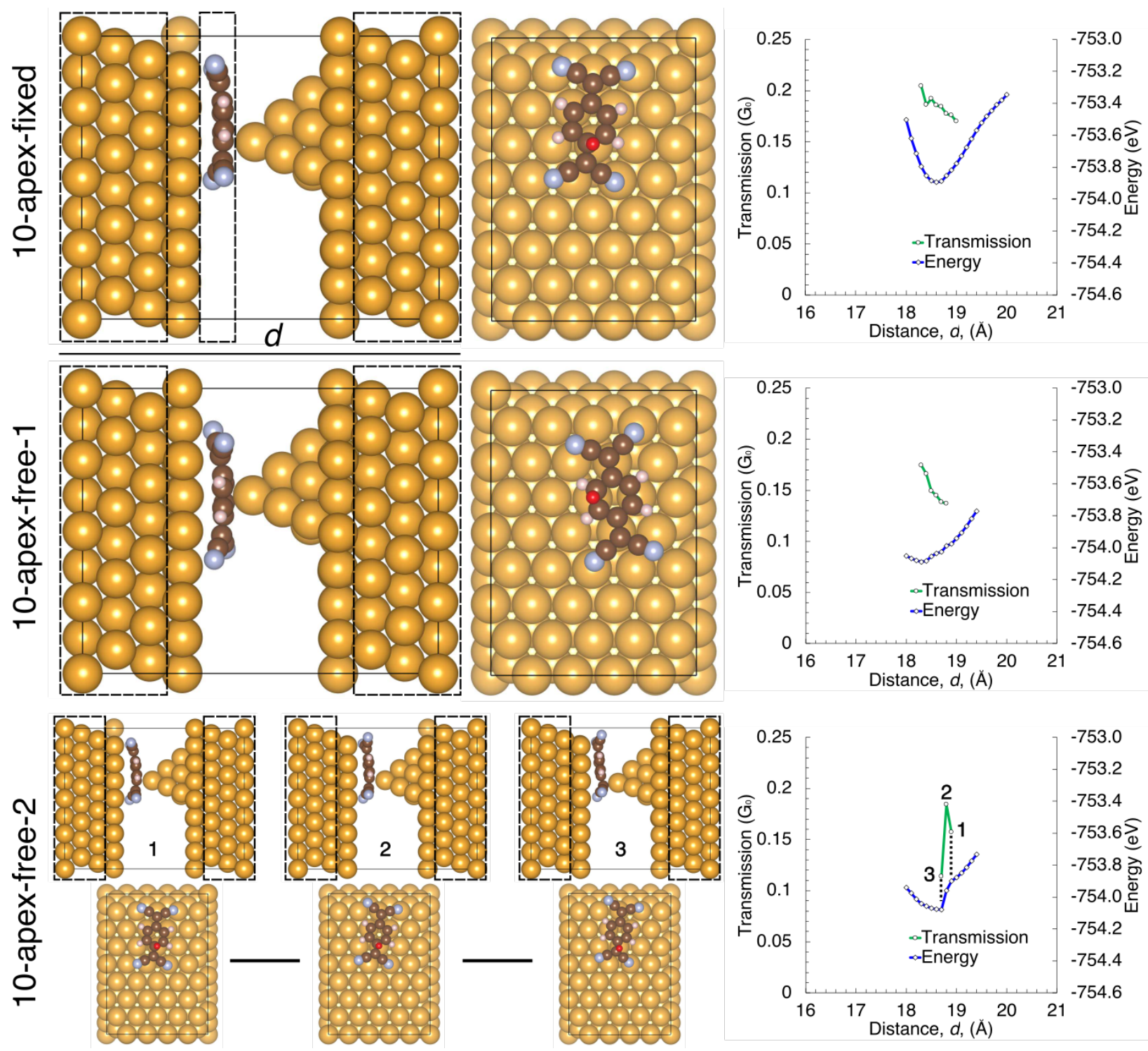

Figure S21. Side views, top views, and plots of energy and low-bias transmission as a function of electrode distance, $d$, of three different series of flat oriented TCNQ molecules. The geometries of the energy minimum are shown for 10-apex-fixed and 10-apex-free-1. Three geometries of interest are shown for 10-apex-free-2. In the top views, the small red atom represents the location of the Au atom tip. Black dotted lines enclose fixed atoms during optimization.

Multiple series of flat geometries were calculated for TCNQ and the results are shown in Figure S21. All systems consist of one flat electrode and one flat electrode with a 10-atom apex. When the TCNQ molecule is kept fixed (10-apex-fixed) throughout $d$ optimization, a $\mathrm{C}$ atom in the benzene ring remains aligned with the apex. At the optimum distance the low-bias conductance is $0.19 \mathrm{G}_{0}$, similar to the result for 1-apex-free for $\mathrm{F}_{4}$ TCNQ $\left(\sim 0.2 \mathrm{G}_{0}\right)$. Two series are shown in which the TCNQ molecule is free to move, 10-apex-free-1 and -2. The initial orientations of the TCNQ molecule on the flat Au electrode are purposefully changed slightly which ultimately leads to different molecule-apex alignment. In 10-apex-free-1, the apex aligns roughly above a center $\mathrm{C}-\mathrm{C}$ bond in the benzene ring, giving a low-bias conductance of $0.17 \mathrm{G}_{0}$ at the minimum 
energy separation. For TCNQ there is small variation between the $\mathrm{C}$ atom and $\mathrm{C}-\mathrm{C}$ bond apex alignments, while for $\mathrm{F}_{4} \mathrm{TCNQ}$ there is a significant difference $\left(\sim 0.1 \mathrm{G}_{0}\right)$ between the two. In 10 -apex-free-2, the apex is initially aligned as in the fixed system (above a $\mathrm{C}$ atom in the benzene ring). Before the optimum $d$ is found, the molecule shifts and the apex aligns above the $\mathrm{C}$ atom just outside the central benzene ring. Prior to the shift (2 in Figure S21) the conductance is $0.18 \mathrm{G}_{0}$, similar to the previous results. After the shift to the outside of the ring (3), the conductance drops to $0.11 \mathrm{G}_{0}$. Since no similar alignment occurred with $\mathrm{F}_{4} \mathrm{TCNQ}$ no comparison between the two could be made. Thus, a discussion of this alignment was left out of the main text.

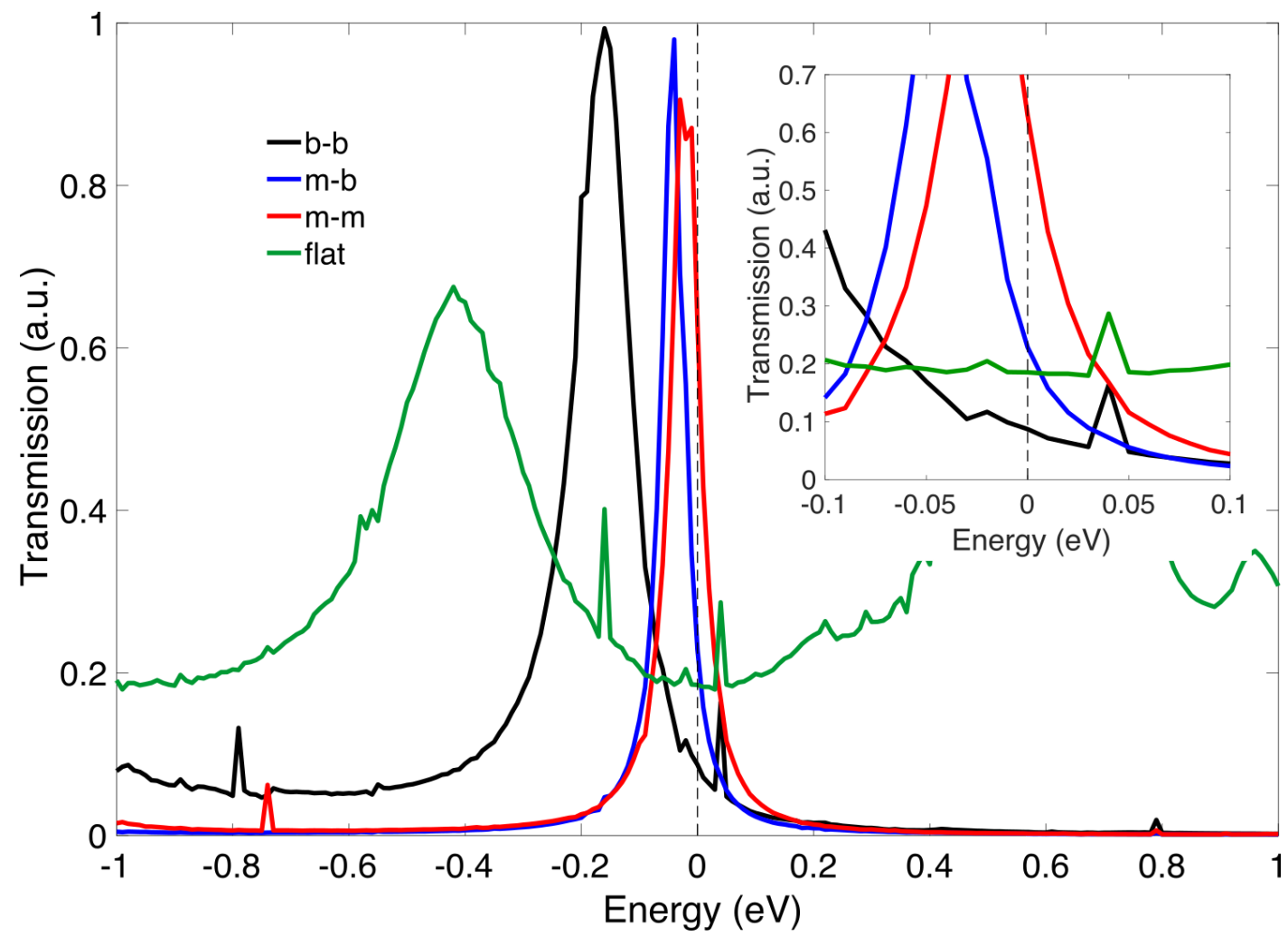

Figure S22. Calculated transmission spectra of a single TCNQ molecule between two Au electrodes in different orientations. The b-b transmission corresponds to the geometry at point 1 in Figure S19 and the flat to 10-apex-fixed in Figure S21.

The transmission spectra in Figure S22 show a similar trend in TCNQ to that of $\mathrm{F}_{4} \mathrm{TCNQ}$, in which the frontier orbital shifts toward the Fermi energy as we change from $b-b$ to $m-b$ to $m-m$. 
d. Calculations of isolated $\mathrm{F}_{4}$ TCNQ and TCNQ molecules

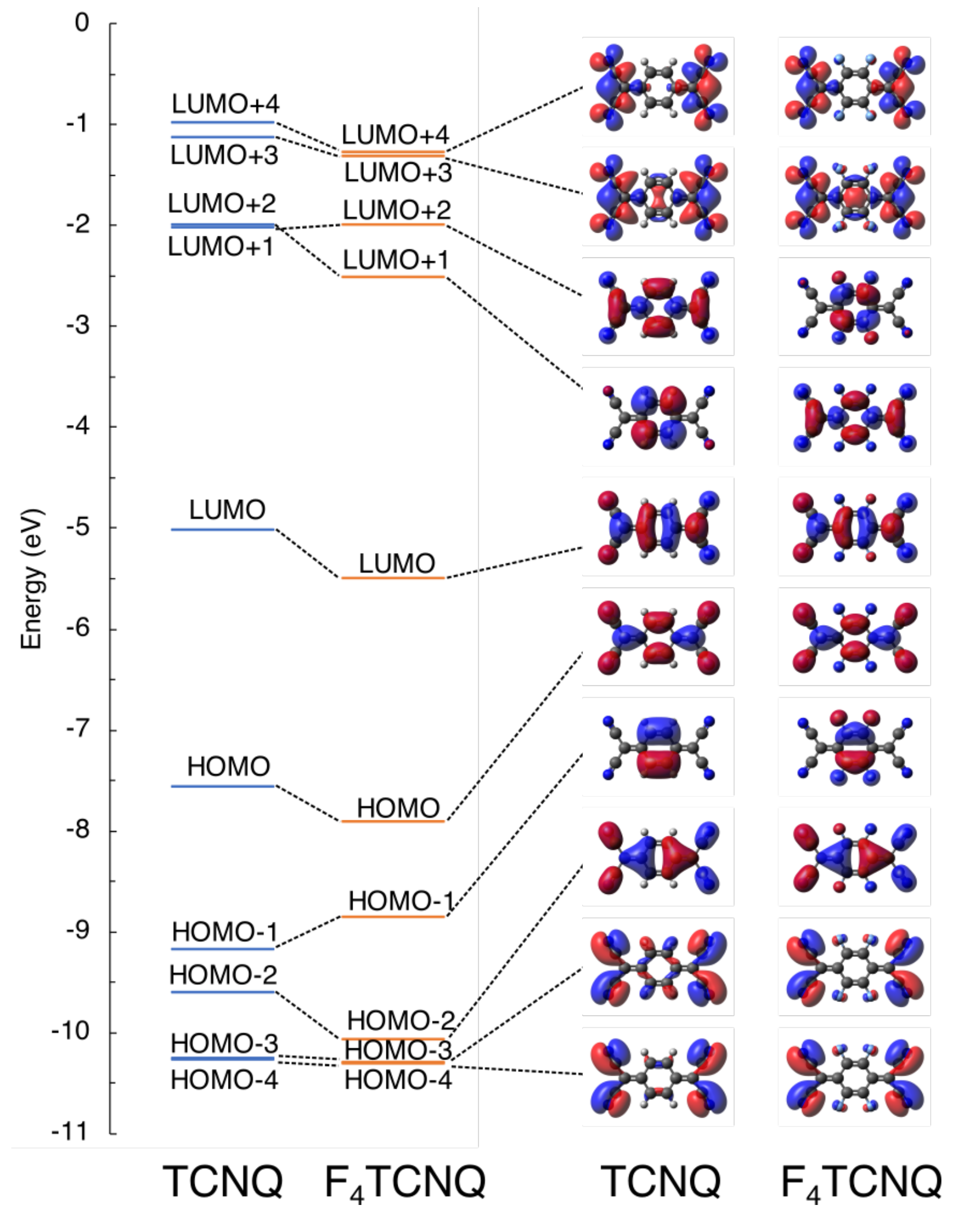

Figure S23. Calculated molecular orbital energies and real space wave functions (HOMO-4 to LUMO+4) of isolated TCNQ and $\mathrm{F}_{4} \mathrm{TCNQ}$. All wave functions are plotted with an isosurface value of 0.02 . 
The molecular orbitals of a single $\mathrm{F}_{4}$ TCNQ molecule were calculated using the Orca program ${ }^{15}$ with the $\mathrm{B}^{2} \mathrm{LYP}^{16}$ functional and a $6-311 \mathrm{G}(\mathrm{d}, \mathrm{p})$ basis set and are shown in Figure S23. In general, there is a lowering in the energies in $\mathrm{F}_{4} \mathrm{TCNQ}$ relative to TCNQ except for the HOMO-1 and LUMO+2.

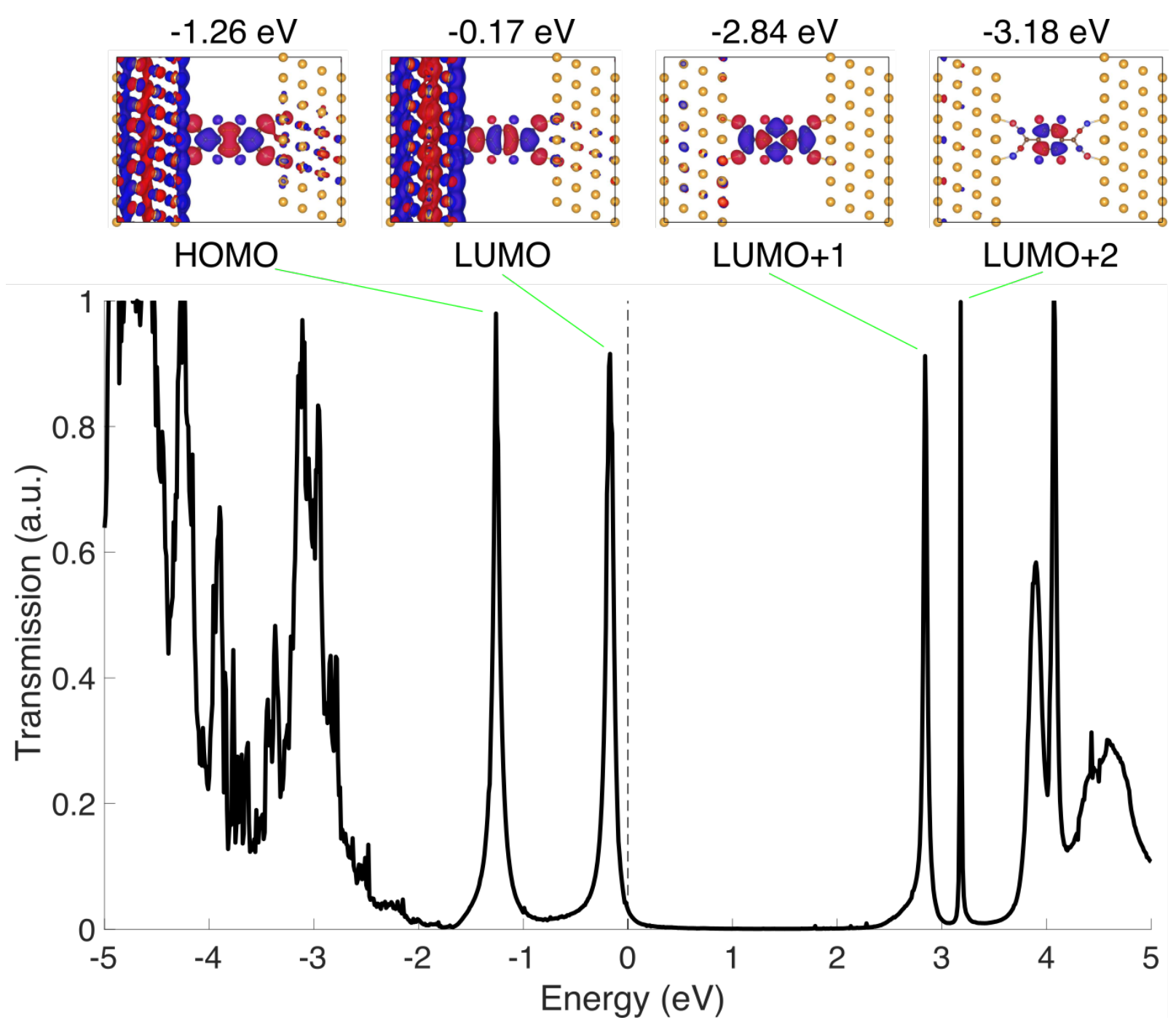

Figure S24. Top: Scattering state wave functions calculated at specified energies. Bottom: Calculated wide energy range transmission spectra for $b-b_{4} F_{4}$ TCNQ. The molecular orbital labels are assigned based on comparison to molecular wave functions calculated with hybrid DFT. 


\section{References:}

1. Cuesta, A.; Kleinert, M.; Kolb, D. M., The adsorption of sulfate and phosphate on $\mathrm{Au}(111)$ and $\mathrm{Au}(100)$ electrodes: an in situ STM study. Phys. Chem. Chem. Phys. 2000, 2, 5684-5690.

2. Le, T. H.; Nafady, A.; Qu, X.; Bond, A. M.; Martin, L. L., Redox and Acid-Base Chemistry of 7,7,8,8-Tetracyanoquinodimethane, 7,7,8,8-Tetracyanoquinodimethane Radical Anion, 7,7,8,8Tetracyanoquinodimethane Dianion, and Dihydro-7,7,8,8-Tetracyanoquinodimethane in Acetonitrile. Anal. Chem. 2012, 84, 2343-2350.

3. Harris, A. R.; Nafady, A.; O'Mullan, A. P.; Bond, A. M., Voltammetric, Spectroscopic, and Microscopic Investigations of Electrocrystallized Forms of Semiconducting AgTCNQ (TCNQ = 7,7,8,8Tetracyanoquinodimethane) Exhibiting Different Morphologies and Colors. Chem. Mater. 2007, 19, 5499-5509.

4. He, Y.; Ye, T.; Borguet, E., The role of hydrophobic chains in self-assembly at electrified interfaces: Observation of potential-induced transformations of two-dimensional crystals of hexadecane by in-situ scanning tunneling microscopy. J. Phys. Chem. B 2002, 106, 11264-11271.

5. Kresse, G.; Furthmuller, J., Efficient Iterative Schemes for Ab Initio Total-Energy Calculations Using a Plane-Wave Basis Set. Phys. Rev. B 1996, 54, 11169-11186.

6. Perdew, J.; Burke, K.; Ernzerhof, M., Generalized gradient approximation made simple. Phys. Rev. Lett. 1996, 77, 3865-3868.

7. Grimme, S.; Antony, J.; Ehrlich, S.; Krieg, H., A consistent and accurate ab initio parametrization of density functional dispersion correction (DFT-D) for the 94 elements H-Pu. J. Chem. Phys. 2010, 132, 154104.

8. Blochl, P. E., Projector augmented-wave method. Phys Rev B Condens Matter 1994, 50, 1795317979.

9. Klimes, J.; Bowler, D.; Michaelides, A., Van der Waals density functionals applied to solids. Phys. Rev. B 2011, 83, 195131.

10. Taylor, J.; Guo, H.; Wang, J., Ab Initio Modeling of Quantum Transport Properties of Molecular Electronic Devices. Phys. Rev. B 2001, 63, 245407.

11. Taylor, J.; Guo, H.; Wang, J., Ab Initio Modeling of Open Systems: Charge Transfer, Electron Conduction, and Molecular Switching of a C-60 Device. Phys. Rev. B 2001, 63, 121104.

12. Romaner, L.; Heimel, G.; Gruber, M.; Bredas, J.; Zojer, E., Stretching and breaking of a molecular junction. Small 2006, 2, 1468-1475.

13. Li, Z.; Kosov, D., Nature of well-defined conductance of amine-anchored molecular junctions: Density functional calculations. Phys. Rev. B 2007, 76, 035415.

14. Bruot, C.; Hihath, J.; Tao, N., Mechanically controlled molecular orbital alignment in single molecule junctions. Nat. Nanotechnol. 2012, 7, 35-40.

15. Neese, F., The ORCA program system. Wiley Interdisciplinary Reviews-Computational Molecular Science 2012, 2, 73-78.

16. Becke, A., Density-functional thermochemistry. III. The role of exact exchange. J. Chem. Phys. 1993, 98, 5648. 